\title{
Reformas estructurales y crecimiento en América Latina: un análisis de sensibilidad
}

\section{Rafael Correa}

Departamento de Economía,

Universidad de

San Francisco de Quito,

Ecuador

rafaelc@andinanet.net
Desde mediados de los años ochenta, la mayoría de los países de América Latina comenzó un profundo proceso de reformas estructurales en la línea del llamado Consenso de Washington. Este artículo pone a prueba la robustez de la evidencia empírica suministrada por varios estudios en favor de las reformas y su positivo impacto en el crecimiento latinoamericano. Los resultados son notables. Ninguna reforma es robusta y positivamente correlacionada con crecimiento, inversión o crecimiento de la productividad en la región, y existe evidencia de que algunas reformas, y particularmente la flexibilización laboral, en realidad pueden estar perjudicando el crecimiento. Los resultados también muestran que los efectos temporales para el período 1987-1995, si —algo— han sido positivos, contradiciendo la generalizada opinión de que el bajo crecimiento latinoamericano, a pesar de las muchas y profundas reformas estructurales, fue producto de un entorno internacional adverso. 
"Los economistas han heredado de las ciencias físicas el mito de que la inferencia científica es objetiva y está libre de prejuicios personales. Esto es un disparate. Todo conocimiento es producto de la convicción humana; dicho en forma más precisa, es producto de la opinión humana”.

LEAMER, 1983

\section{I}

\section{Introducción}

Desde mediados del decenio de 1980, en las secuelas de la crisis de la deuda, la mayoría de los países latinoamericanos comenzaron un proceso acelerado de liberalización e internacionalización de sus economías en consonancia con el denominado Consenso de Washington. Sin embargo, pese a las múltiples y profundas reformas estructurales y no obstante la disminución de 1.8\% del PIB per cápita durante 1981-1985, el crecimiento económico per cápita durante 1986-1995 fue sólo de $1.2 \%$, mientras que durante los años sesenta y setenta la región llegó a tasas medias de $2.5 \%$ y $2.4 \%$, respectivamente. ${ }^{1}$

Pese a este hecho, varios estudios empíricos han demostrado que las reformas estructurales han tenido un efecto positivo sobre el crecimiento regional, aduciendo que el mal desempeño económico se ha debido a la ausencia de reformas todavía más radicales, a la falta de complementariedad de las políticas ${ }^{2}$ y a factores internacionales.

Concretamente, el Banco Interamericano de Desarrollo (BID, 1997), usando índices de políticas representativos de cinco áreas de reforma y modelos de datos de panel, estimó que las reformas habían contribuido en $1.9 \%$ al crecimiento permanente de la región y que la profundización de las mismas podía contribuir aún entre $1.2 \%$ y $1.6 \%$. Según el informe del BID, las reformas incrementaron el crecimiento sobre todo gracias a la mejor asignación de recursos, factor que había contribuido en $1.7 \%$ al crecimiento permanente, y también tuvieron efectos positivos pero modestos sobre la inversión. El informe observó asimimo que los efectos de las reformas, en contra de lo que suele creerse, habían sido rápidos, y en consecuencia recomendó enfáticamente acelerar el proceso reformador.

\footnotetext{
${ }^{1}$ Datos del Banco Mundial para 19 economías latinoamericanas.

${ }^{2}$ La complementariedad de las políticas significa, en esencia, que el efecto positivo de las reformas está condicionado a la heterogeneidad de los sectores. Por ende, aunque el país haya hecho reformas económicas de gran envergadura, su efecto sobre el crecimiento se esfumará si el nivel de reformas en las distintas áreas es demasiado dispar.
}

Los índices de políticas utilizados por el BID han sido empleados también en regresiones de crecimiento de datos de panel por Barrera y Lora (1997) y Fernández-Arias y Montiel (1997), autores que llegaron a conclusiones similares a las de ese organismo. ${ }^{3}$ Sin embargo, Aziz y Wescott (1997), en un estudio efectuado a nivel mundial usando datos de corte transversal y tres índices de políticas, encontraron que ninguna reforma se correlacionaba robustamente con el crecimiento. Empero, sostuvieron que este resultado se debía a la disparidad de las reformas entre diferentes sectores y hallaron que la complementariedad de las políticas, representada por la diferencia entre una medida de tendencia central y una medida de dispersión de los índices de políticas, estaba correlacionada en forma robusta y positiva con el crecimiento. Por último, Easterly, Loayza y Montiel (1997), utilizando seis índices de políticas y modelos dinámicos de datos de panel, concluyeron que las reformas y su impacto sobre la recuperación del crecimiento en América Latina habían sido un logro impresionante, y explicaban, en parte, el bajo crecimiento económico de la región por la intervención de factores internacionales adversos.

Este estudio pone a prueba la robustez de la correlación entre las reformas estructurales y el crecimiento económico latinoamericano o sus fuentes. De igual manera, pone a prueba la robustez de la correlación entre la complementariedad de políticas y el crecimiento, y si un entorno internacional negativo perjudicó el crecimiento regional. La investigación utiliza los mismos índices de políticas estructurales y la misma estructura de datos de panel que los empleados por el Banco Interamericano de Desarrollo (BID, 1997). Por ende, la muestra se compone de 19 países latinoamericanos y abarca cuatro períodos trienales: 1984-1986,

\footnotetext{
${ }^{3}$ De hecho, el Informe 1997 del Banco Interamericano de Desarro1lo (BID, 1997, capítulo II) reproduce las observaciones de Barrera y Lora (1997)
} 
1987-1989, 1990-1992 y 1993-1995. Los países incluidos en el estudio son Argentina, Bolivia, Brasil, Chile, Colombia, Costa Rica, Ecuador, El Salvador, Guatemala, Honduras, Jamaica, México, Nicaragua, Paraguay, Perú, República Dominicana, Trinidad y Tabago, Uruguay y Venezuela. El artículo usa modelos de datos de panel y el procedimiento de estimación controla los efectos fijos y problemas de endogeneidad y utiliza eficientes estimadores generalizados de momentos en dos etapas. La prueba de robustez es el análisis de límites extremos sugerido por Leamer (1983) y empleado en regresiones de crecimiento por Levine y Renelt (1992).
El artículo se organiza como sigue. La sección II describe los índices de políticas estructurales. La sección III destaca la importancia de controlar los efectos y tendencias temporales del panel en estudio, ilustra las ventajas de usar modelos de datos de panel en las regresiones de crecimiento y explica el procedimiento de estimación. La sección IV examina la metodología del análisis de límites extremos. La sección V presenta los datos y modelos que se utilizarán para realizar tal análisis y las diferentes clasificaciones de las variables. La sección VI reporta los resultados econométricos y, por último, la sección VII presenta las conclusiones.

\section{II}

\section{Medición de las reformas estructurales}

Uno de los obstáculos principales para evaluar cuantitativamente las reformas estructurales en América Latina era la falta de una adecuada metodología para medir el proceso reformador. Para cuantificar de manera global dicho proceso en la región, Lora (1997) creó el "índice de política estructural" (sPI, por su sigla en inglés). El SPI es la media aritmética de cinco índices sectoriales que representan: liberalización comercial (TRADE), neutralidad tributaria (TAX), liberalización financiera (FIN), privatización (PRIV) y flexibilización laboral (LAB). A su vez, cada índice sectorial es la media aritmética de una o más variables de política, cuyos valores están normalizados con respecto a la peor y la mejor observación de esa variable en toda la muestra de países y años (véase el apéndice A). En consecuencia, cada índice puede moverse en un rango de valores de 0 a 1 de manera tal que mientras más cercano esté a 1 , más neutral será la política económica del país. De esta forma, el sPI y sus cinco componentes tratan de ser medidas del nivel de políticas de libre mercado. Por último, cabe destacar que los índices están compuestos explícitamente por variables de política, con lo que se evita una de las deficiencias principales de varios estudios previos, que utilizaron los objetivos buscados por las reformas como medida de las mismas. ${ }^{4}$

\footnotetext{
${ }^{4}$ Varios estudios usan como medida de las reformas sus presuntos resultados en vez de variables de política. Por ejemplo, Easterly, Loayza y Montiel (1997) señalaron explícitamente que sus
}

Usando el sPI, el cuadro 1 muestra las rápidas y profundas reformas estructurales implantadas en América Latina durante 1985-1995 y la trayectoria y clasificación de los distintos países. En diez años, el SPI promedio de la región subió de 0.345 a 0.621 . Para ilustrar la rapidez con que han ocurrido las reformas en algunos países, nótese que en 1985 y 1990 Chile era el mejor ejemplo de una economía de libre mercado y que en sólo cinco años bajó al noveno lugar. El proceso ha sido tan generalizado que el SPI más bajo en 1995 —el de Venezuela— sería el tercero más alto en 1985.

Los diagramas Box-and-Whisker (gráfico 1) muestran el comportamiento de cada área de reforma. Las políticas más neutrales — representadas en los diagramas por medianas más altas y menor dispersión - se dan en el comercio exterior y el sector financiero latinoamericanos. Sin embargo, todas las áreas de reforma muestran medianas sustancialmente más altas que en 1985, aunque la privatización y la flexibilización laboral en 1995 exhiben todavía niveles muy dispares entre los diferentes países.

indicadores de reformas 'debían moverse en el sentido cualitativo asociado con la reforma... y debían explicar el crecimiento' (Easterly, Loayza y Montiel, 1997, p. 293). Así, el efecto positivo de las reformas sobre el crecimiento está prácticamente garantizado. 
América Latina y el Caribe: Indice de política estructural (SPI)

\begin{tabular}{|c|c|c|c|c|c|c|}
\hline & \multicolumn{2}{|c|}{1985} & \multicolumn{2}{|c|}{1990} & \multicolumn{2}{|c|}{1995} \\
\hline & SPI & $\overline{\text { Lugar }}$ & SPI & $\overline{\text { Lugar }}$ & SPI & Lugar \\
\hline Argentina & 0.367 & 4 & 0.476 & 12 & 0.679 & 5 \\
\hline Bolivia & 0.343 & 6 & 0.548 & 5 & 0.721 & 1 \\
\hline Brasil & 0.348 & 5 & 0.512 & 8 & 0.584 & 13 \\
\hline Chile & 0.489 & 1 & 0.596 & 1 & 0.628 & 9 \\
\hline Colombia & 0.443 & 3 & 0.549 & 4 & 0.590 & 12 \\
\hline Costa Rica & 0.309 & 10 & 0.500 & 10 & 0.512 & 18 \\
\hline República Dominicana & & & 0.361 & 17 & 0.638 & 8 \\
\hline Ecuador & 0.325 & 9 & 0.357 & 18 & 0.580 & 14 \\
\hline El Salvador & & & 0.532 & 7 & 0.671 & 6 \\
\hline Guatemala & 0.309 & 10 & 0.438 & 14 & 0.596 & 11 \\
\hline Honduras & & & 0.450 & 13 & 0.548 & 17 \\
\hline Jamaica & & & 0.573 & 3 & 0.684 & 4 \\
\hline México & 0.328 & 8 & 0.498 & 11 & 0.563 & 16 \\
\hline Nicaragua & 0.216 & 14 & 0.391 & 15 & 0.643 & 7 \\
\hline Paraguay & 0.336 & 7 & 0.548 & 5 & 0.625 & 10 \\
\hline Perú & 0.232 & 13 & 0.252 & 19 & 0.712 & 3 \\
\hline Trinidad y Tabago & & & 0.589 & 2 & 0.715 & 2 \\
\hline Uruguay & 0.486 & 2 & 0.511 & 9 & 0.573 & 15 \\
\hline Venezuela & 0.304 & 12 & 0.364 & 16 & 0.457 & 19 \\
\hline Promedio & 0.345 & & 0.479 & & 0.621 & \\
\hline
\end{tabular}

GRAFICO 1

América Latina y el Caribe: Evolución de cada área de reforma

1985

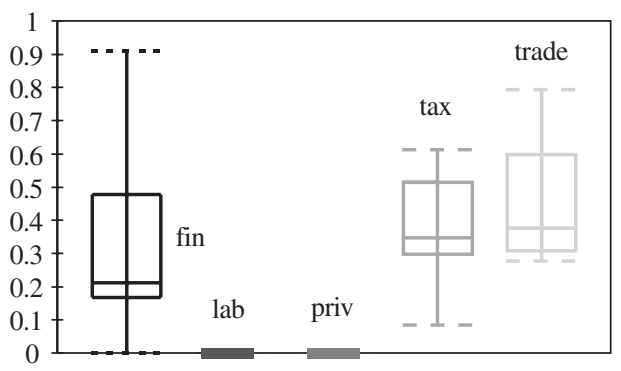

1990

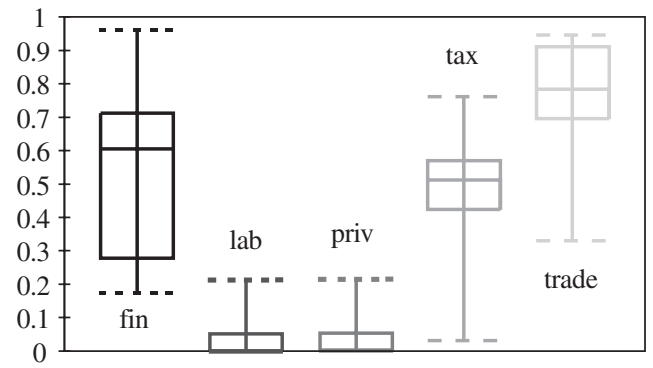

1995

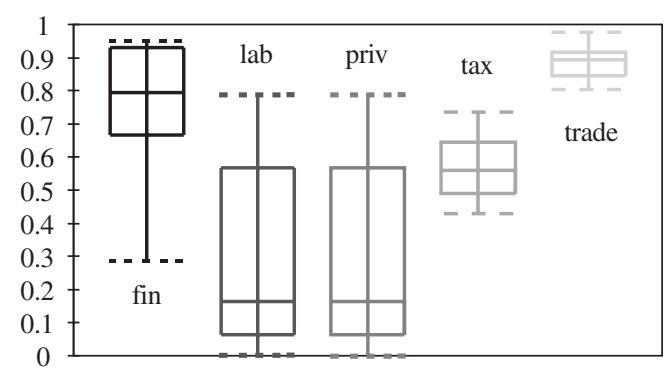




\section{III}

\section{Aspectos econométricos}

GRAFICO 2

Crecimiento, índice de política estructural (SPI) y tasa de interés

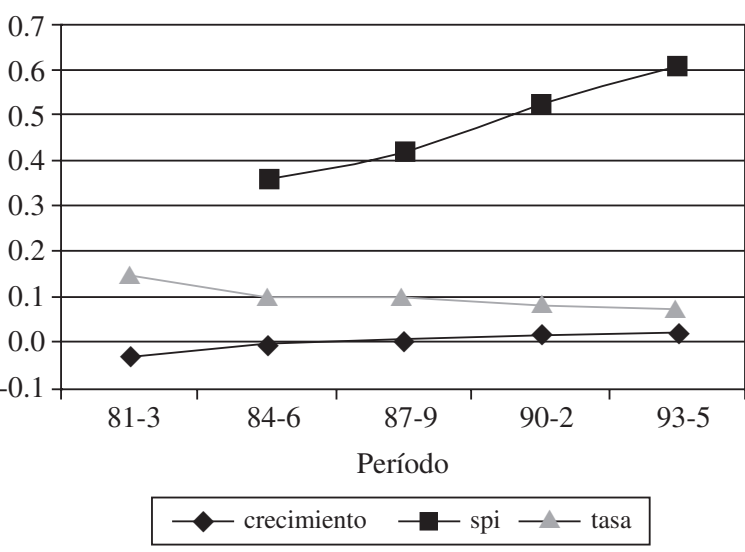

1. La tendencia temporal del crecimiento, la tasa de interés y el índice de política estructural

Aunque el crecimiento per cápita regional en 19851995 fue bajo comparado con el de los años sesenta y setenta, presentó una tendencia ascendente a partir de la crisis de la deuda de comienzos de los años ochenta (gráfico 2). Esta tendencia fue coincidente con la evolución del sPi para toda América Latina, pero también fue un perfecto reflejo del comportamiento decreciente de la tasa de interés internacional. Dicha tasa es una variable decisiva para el desempeño económico latinoamericano, puesto que tiene un impacto considerable sobre el servicio de la deuda externa y la entrada de capitales a la región. El gráfico muestra también estas tres variables en diferencias para así eliminar las tendencias temporales. Dada la breve dimensión temporal de nuestro panel, la simple inspección sería en general una manera arriesgada de determinar cualquier relación entre las variables. Sin embargo, parece bastante claro que los cambios de la tasa de crecimiento siguen siendo un perfecto reflejo de los cambios de la tasa de interés.

La situación descrita resalta dos problemas simultáneos que deben ser considerados. El primero es la influencia de factores internacionales que dificultan saber si el crecimiento se incrementó debido a mejores políticas o, por ejemplo, debido a una tasa de interés internacional decreciente. El segundo proble-

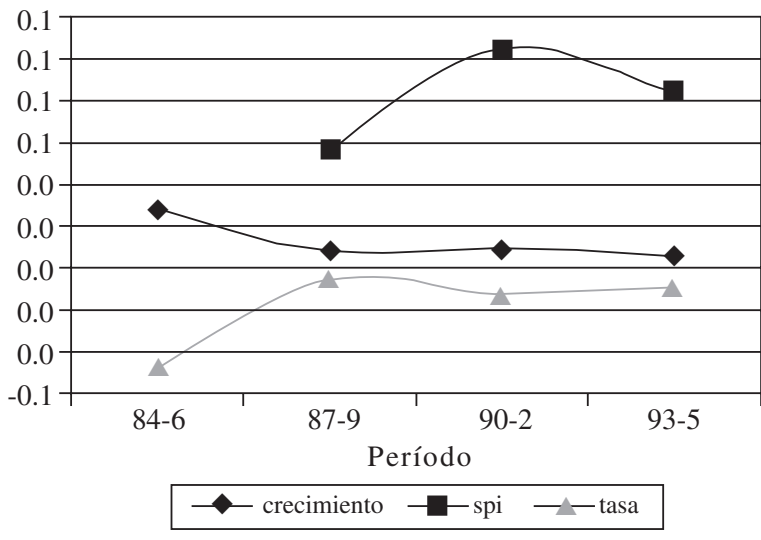

ma es el riesgo de obtener una correlación espuria debido a la tendencia temporal que muestran el crecimiento y las reformas estructurales. Para resolver en parte estos problemas, el procedimiento de estimación debe controlar la situación del entorno económico internacional y la tendencia temporal en la tasa de crecimiento y en las variables explicativas.

\section{Datos de panel y regresiones de crecimiento}

Varios autores han destacado la importancia de los datos de panel para las regresiones de crecimiento. ${ }^{5} \mathrm{La}$ estructura de panel tiene ventajas fundamentales con respecto a las regresiones de crecimiento de corte transversal, pues ofrece más grados de libertad, instrumentos para controlar problemas de endogeneidad y mecanismos para controlar los efectos individuales y temporales.

Mayores grados de libertad resultan decisivos cuando estamos interesados en una región o subconjunto de países con características similares. La metodología de corte transversal normalmente emplea variables ficticias regionales para permitir modelos con diferentes términos independientes entre regiones. Sin embargo, con este procedimiento se debería aceptar que el efecto marginal de toda variable explicativa es

\footnotetext{
${ }^{5}$ Véanse Islam (1995) y Caselli, Esquivel y Lefort (1996).
} 
el mismo en un país de la Organización de Cooperación y Desarrollo Económicos (OCDE), en uno de Africa o en uno de América Latina. De esta manera, el mayor grado de libertad que ofrecen los datos de panel facilita el estudio de sólo un subconjunto de países, lo que hace más plausible el supuesto implícito de homogeneidad de coeficientes.

Otra gran ventaja de los datos de panel obedece a su dimensión temporal, que provee instrumentos válidos por medio de los valores retardados de las variables explicativas. Dado que casi toda variable explicativa de las regresiones de crecimiento podría considerarse endógena, la disponibilidad de instrumentos permite obtener estimaciones consistentes.

Por último, la ventaja fundamental es que los modelos de datos de panel son la única manera de controlar los efectos individuales y temporales. Cuando no se controlan estos efectos fijos surge el problema de variables omitidas y los coeficientes estimados resultarán sesgados, a menos que utilicemos el supuesto inverosímil de ortogonalidad entre los efectos y las variables explicativas. La consideración de los efectos temporales tiene especial importancia cuando se trata de países subdesarrollados, cuyas economías dependen en gran medida del entorno económico internacional, como se ilustró en la sección precedente. Por otra parte, también reviste importancia primordial el control de los efectos individuales, porque difícilmente podemos concebir variables que expliquen el crecimiento y no estén correlacionadas con las capacidades económicas y sociales no observables de cada país. Más aun, cuando utilizamos como variable explicativa los valores retardados de la variable dependiente, como se hace en la mayoría de los estudios de corte transversal, el supuesto inverosímil de ortogonalidad necesariamente es violado, como señalaron acertadamente Caselli, Esquivel y Lefort (1996, p. 367).

\section{Procedimiento de estimación}

Los modelos de datos de panel que van a estimarse (véase el apéndice B) tienen la siguiente forma general: 6

$$
\begin{gathered}
y_{i t}=\mathrm{x}_{\mathrm{it}}{ }^{\prime} \uparrow+\eta_{t}+\mapsto_{i}+v_{i t} \\
\mathrm{i}=1,2, \ldots, \mathrm{N} . \quad \mathrm{t}=1,2, \ldots, \mathrm{T} .
\end{gathered}
$$

\footnotetext{
${ }^{6}$ Aunque en realidad usamos paneles desbalanceados, para simplificar la notación en esta sección, suponemos que se utilizan paneles balanceados.
}

donde $\mathrm{x}_{\text {it }}$ es el vector $\mathrm{K}$ x 1 de variables explicativas que pueden contener la variable dependiente retardada en un período, regresores endógenos pero débilmente exógenos $\left(\mathrm{E}\left[\mathrm{x}^{1}{ }_{\text {it }} v_{i s}\right]=0\right.$ para todo $\left.\mathrm{t}<\mathrm{s}\right)$ y regresores estrictamente exógenos $\left(\mathrm{E}\left[\mathrm{x}^{2}{ }_{\text {it }} v_{i s}\right]=0\right.$ para todo t,s); $\beta$ es un vector $K \times 1$ de constantes, $\eta_{t} \mathrm{y} \mapsto \mapsto_{i}$ son los efectos temporales e individuales no observables, respectivamente, y $v_{i t}$ es una perturbación aleatoria no correlacionada serialmente.

Las especificaciones son modelos de efectos fijos en el sentido de que $\eta_{t} \mathrm{y} \mapsto_{i}$ son constantes fijas y no una perturbación aleatoria que pueda incorporarse en el término de error. La especificación de efectos fijos es la adecuada puesto que nos vamos a centrar en un conjunto específico de países durante un período de tiempo determinado y, por lo tanto, los efectos no se han extraído al azar de una población numerosa.

Siguiendo a Anderson y Hsiao (1981), controlamos los efectos individuales poniendo [1] en primeras diferencias. ${ }^{7}$ Nótese que esta transformación elimina también cualquier tendencia temporal de las variables. ${ }^{8}$ Para controlar los efectos temporales, éstos serán estimados utilizando variables ficticias que representan los diferentes períodos de tiempo. Por último, obsérvese que debido a perfecta multicolinealidad la especificación de efectos fijos impide la identificación de los coeficientes de cualquier variable temporal o individualmente invariante. De esta forma, los modelos reales que efectivamente van a estimarse poseen la forma general:

$$
\begin{aligned}
& \lambda y_{i t}=\lambda \mathrm{x}_{\mathrm{it}}{ }^{\prime} \uparrow+\mathrm{d}_{\mathrm{t}}{ }^{\prime} \phi+\lambda v_{i t} \\
& \mathrm{i}=1,2, \ldots, \mathrm{N} . \quad \mathrm{t}=1,2, \ldots, \mathrm{T} \text {. }
\end{aligned}
$$

donde $d_{t}$ es un vector $(T-2)$ x 1 de variables ficticias que representan los períodos $3,4, \ldots ., \mathrm{y} \phi$ es un vector $(T-2)$ x 1 de constantes. Bajo el supuesto de que $v_{i t}$ no está serialmente correlacionada, cualquier valor de $y_{i t}$

\footnotetext{
${ }^{7}$ Nótese que si $\mathrm{x}_{\mathrm{it}}$ contiene cualquier retardo de $y_{i t}$, el estimador intragrupos es inconsistente porque $\left(y_{i t}-s-\bar{y}_{i .}\right)$ y $\left(v_{i t}-\bar{v}_{i .}\right)$ están correlacionados puesto que el promedio de esta última contiene $v_{i t-s}$. ${ }^{8}$ El modelo en primeras diferencias puede exacerbar la inconsistencia generada por los errores de medición cuando la variable explicativa tiene una tendencia temporal, como en el caso de los índices de políticas. No obstante, cabe señalar que el sesgo siempre tiene el signo contrario al del coeficiente ("sesgo de atenuación"), de modo que los errores de medición pueden generar falta de significación estadística, pero no coeficientes con signo errado. Sin embargo, en general el uso de variables de política - no de agregados macroeconómicos- en la construcción de los índices minimiza el riesgo de incurrir en errores de medición.
} 
o de $\mathrm{x}^{1}{ }_{\text {it }}$ retardado dos o más períodos es un instrumento válido para la ecuación en primeras diferencias y, por ende, con $T \geq 3$ podemos hacer una estimación consistente de [2]. ${ }^{9}$

Para ganar eficiencia, usamos el estimador generalizado de momentos (GMM) en dos etapas propuesto por Arellano y Bond (1991). Aprovechando un conjunto de condiciones de ortogonalidad derivadas de los supuestos sobre el término de error y los regresores, aplicamos la matriz instrumental $W=(Z, d)$, donde $d$ es la matriz de variables ficticias, $Z=\left(z_{1}{ }^{\prime}, \ldots, Z_{N}{ }^{\prime}\right)^{\prime}, y$ $z_{i}=\operatorname{diag}\left(y_{i 1} ; \ldots, y_{i t-2} ; x_{i 1}^{l}, \ldots, x_{i t-2}^{l} ; \mathrm{D} x_{i 3}^{2}, \ldots, \mathrm{D}^{2}{ }_{i t}{ }^{\prime}\right)$.
La estimación se hace en dos etapas. Primero, se realiza una estimación de [2] usando la matriz de instrumentos $W \cdot{ }^{10}$ Luego, con los residuos de esta primera estimación se estima una matriz de covarianza, y se realiza nuevamente una estimación de [2] usando la matriz de instrumentos $W$ y la matriz de covarianza anteriormente estimada. Por último, efectuamos un test de Sargan de restricciones de sobreidentificación para verificar la validez de los instrumentos, y calculamos el número de condición de la matriz de variables explicativas en [2], para verificar si existen problemas de multicolinealidad.

\section{IV}

\section{Análisis de límites extremos}

Para verificar la robustez de la correlación entre reformas estructurales y crecimiento, aplicamos una variante del análisis de límites extremos sugerido por Leamer (1983) y empleado en regresiones de crecimiento por Levine y Renelt (1992). El análisis de límites extremos es una búsqueda formal de diferentes especificaciones mediante la variación sistemática del conjunto de información condicionante, para así estimar la dimensión de la incertidumbre sobre el verdadero modelo.

Las variables explicativas se clasifican como: la variable central, las variables libres $(I)$ y las variables dudosas $(D)$. La variable central es aquélla cuyo coeficiente $\left(\uparrow_{F}\right)$ nos interesa. Las variables $I$ y la central están siempre en el conjunto de información, mientras que las variables $D$ pueden combinarse de cualquier manera lineal. ${ }^{11}$

Para un nivel de confianza dado, el límite extremo superior (inferior) de $\uparrow_{F}$ es la estimación correspondiente al máximo límite superior (mínimo límite inferior) de todos los intervalos de confianza de las diferentes estimaciones de $\uparrow_{F}$. El vínculo entre la variable

\footnotetext{
${ }^{9}$ Nótese que si tenemos $y_{\mathrm{i}, \mathrm{t}-1}$ en el lado derecho de [1], existe siempre endogeneidad de regresores en [2], dado que $\mathrm{E}\left[\Delta y_{i t-1} \Delta v_{i t}\right] \neq 0$ debido al hecho de que $y_{i t-1}$ y $v_{i t-I}$ están correlacionados.

${ }^{10}$ En esta primera etapa, Arellano y Bond (1991) realizan la estimación usando una matriz de ponderación $H i$ cuadrada y de dimensión $(T-1)$ con doses en la diagonal principal, menos unos en las subdiagonales principales y ceros en caso contrario, que representa el supuesto provisional de que $\mathrm{v}_{\mathrm{i}, \mathrm{t}}$ es homocedástica. Dado que toda matriz definida positiva produce una estimación consistente, en vez de $H_{i}$ estamos utilizando la matriz identidad.

${ }^{11} \mathrm{Sin}$ embargo, habitualmente sólo se aplican restricciones de exclusión a las variables $D$.
}

central y la variable dependiente se considera robusto si $\uparrow_{F}$ permanece significativo y con el mismo signo en los límites extremos. De otro modo, el vínculo se define como frágil.

El análisis de límites extremos es particularmente pertinente cuando nos ocupamos de modelos ad hoc que no están avalados por una teoría bien fundamentada y, por consiguiente, tienen una mayor incertidumbre acerca del modelo verdadero. Sin embargo, el análisis de límites extremos ha recibido críticas importantes, concretamente sobre el criterio para definir una variable como frágil, por el riesgo de introducir multicolinealidad, y por el riesgo de omisión de variables relevantes. Sala-i-Martin (1997) sostiene que con el análisis de límites extremos ninguna variable será robusta, puesto que basta con hallar una excepción en que $\widehat{I}_{F}$ se torne insignificante para considerar que la variable central es frágil. El problema con este argumento es que lo opuesto también es cierto: la estimación de diferentes especificaciones ad hoc suele dar un modelo donde $\uparrow_{F}$ es significativo y del signo deseado. Por lo tanto, lo que en el fondo se debate es cuál es el número de éxitos y de fracasos en materia de significación o signo que deberían considerarse para determinar si una variable es robusta o frágil. Sala-iMartin responde esta pregunta construyendo una distribución empírica de probabilidad para $\beta_{F}$ Dados los problemas prácticos que plantea tal análisis, ${ }^{12}$ la

\footnotetext{
${ }^{12}$ Para construir la distribución empírica de probabilidad para $B_{F}$, Sala-i-Martin realizó dos millones de regresiones.
} 
pregunta de "cuánto es suficiente" carece de una respuesta objetiva. Por ejemplo, en el caso de reformas estructurales que cambian países enteros, ¿cuántas excepciones deberíamos permitir para concluir que la evidencia es robusta o frágil? No obstante, para abordar en parte esta crítica procuramos ser lo más descriptivos posibles al dar a conocer los resultados del análisis de límites extremos. Por consiguiente, nuestro análisis de límites extremos reportará los límites extremos; el valor y la significación estadística del coeficiente central en la mejor especificación seleccionada según el criterio de información de Akaike (AIC) y la proporción de coeficientes positivos (negativos) y significativos (no significativos) encontrados para cada variable central.

Otra crítica de peso al análisis de límites extremos es que éste puede introducir variables irrelevantes correlacionadas con el resto de las variables explicativas, inflando con ello los errores estándares de los coeficientes estimados. Sin embargo, mientras los canales causales no sean claros, un problema de multicolinealidad puede significar simplemente que la evidencia es débil (Leamer, 1983, p. 34). No obstante, como regla de decisión para controlar este problema eliminaremos toda variable dudosa $(D)$ con un coeficiente de correlación mayor que 0.5 con respecto a las variables centrales, y jamás incluiremos en los modelos variables $D$ con un coeficiente de correlación mutuo mayor que 0.5 . Por último, conforme a lo mencionado en la sección precedente, en todas las regresiones verificaremos multicolinealidad usando el número de condición de la matriz de regresores. De esta manera, el análisis de límites extremos no considerará ningún modelo con un número de condición mayor que 20 .

Finalmente, la crítica fundamental al análisis de límites extremos es que puede omitir variables relevantes, lo que hace que las estimaciones de $\beta_{F}$ y de $\operatorname{Var}\left(\beta_{F}\right)$ sean sesgadas. Sin embargo, esta crítica es sólo un recordatorio de la debilidad de las inferencias extraídas de modelos ad hoc, y mientras se usen modelos plausibles, la metodología ilustra este problema en vez de causarlo. No obstante, dado que la mejor manera de minimizar el riesgo de omisión de variables relevantes es basarse en una teoría bien establecida, el punto de partida de nuestro análisis será el Modelo Aumentado de Solow propuesto por Mankiw, Romer y Weil (1992), y las variables I de las regresiones de crecimiento serán una aplicación empírica de este modelo.

\section{V}

\section{Datos, modelos y clasificación de las variables}

\section{El punto de partida: el Modelo Aumentado de Solow}

Comenzamos nuestro análisis estimando el modelo

$$
\begin{aligned}
& \lambda \ln G D P_{i t}=\mu+(\rho-1) \ln G D P_{i, t-1}+\uparrow_{1} \ln I N V_{i, t} \\
& +\downarrow_{2} \ln P R O T_{i, t}+\downarrow_{3} \ln P O P_{i, t}+\eta_{t}+\mapsto_{i}+v_{i t}
\end{aligned}
$$

El modelo representa una aproximación empírica del Modelo Aumentado de Solow propuesto por Mankiw, Romer y Weil (1992), donde GDP es el ingreso per cápita; PROT es la tasa de crecimiento de la ingesta media diaria de proteínas y se usa como aproximación a la tasa de acumulación de capital humano; ${ }^{13}$ INV re-

\footnotetext{
13 Para representar la acumulación de capital humano, elegimos una variable nutricional (PROT) en vez de los indicadores de logro escolar usados por la mayoría de los estudios empíricos del crecimiento. Esto obedece a dos razones: primero, cuando utilizamos los indicadores de logro escolar, los factores de calidad no se conside-
}

presenta el coeficiente de inversión y se usa como aproximación a la tasa de acumulación de capital físico, y POP es el crecimiento de la población. Tomando primeras diferencias para controlar los efectos fijos individuales, utilizando variables ficticias para controlar los efectos temporales y manejando términos con la variable dependiente, el modelo puede estimarse como

$$
\begin{aligned}
& \lambda \operatorname{lnGDP}{ }_{i t}=\rho \lambda \operatorname{lnGDP}_{\mathrm{i}, \mathrm{t}-1}+\downarrow_{1} \lambda \operatorname{lnINV}_{\mathrm{i}, \mathrm{t}} \\
& +\uparrow_{2} \lambda \ln \operatorname{lnOT}_{\mathrm{i}, \mathrm{t}}+\uparrow_{3} \lambda \ln \operatorname{lnP}_{\mathrm{i}, \mathrm{t}} \\
& +\Sigma_{j} \phi^{j} d_{t}^{j}+\lambda v_{i t}
\end{aligned}
$$

donde $d^{j}$ son las variables ficticias y $j=3, \ldots, T$.

\footnotetext{
ran, aunque este hecho es crucial para obtener indicadores escolares significativos en los países en desarrollo. Segundo, cuando usamos indicadores como años promedio de escolaridad o tasas de matrícula, jamás queda claro si representan el nivel o tasa de acumulación de capital humano.
} 
Nótese que en el modelo de Solow las únicas fuentes de crecimiento son la acumulación de factores y el crecimiento no observable de la productividad total de los factores $(g)$. Por este motivo, nuestro análisis usa tres diferentes variables dependientes como aproximación al crecimiento y sus fuentes: $\Delta \operatorname{lnGDP}$ que representa el crecimiento, lnINV que representa la acumulación de factores, ${ }^{14}$ y RES, los residuos de nuestro modelo de Solow estimado, como aproximación de la no observable $g$.

\section{Datos y clasificación de las variables}

$\mathrm{El}$ apéndice $\mathrm{C}$ muestra la lista de las variables empleadas en este estudio y describe cada una con su fuente respectiva. La principal fuente de los datos es el Banco Interamericano de Desarrollo.

El artículo examina seis variables centrales en nuestro análisis de límites extremos: los cinco índices de reformas - liberalización comercial (TRADE), neutralidad tributaria (TAX), liberalización financiera (FIN), privatización (PRIV) y flexibilización laboral (LAB)—; la media aritmética de los índices (SPI); su desviación estándar (SIGMA) y la complementariedad de las políticas representada por SPI-SIGMA (POLCOM).

El modelo de Solow establece las variables que en teoría deben incluirse siempre en las regresiones de crecimiento. Por lo tanto, cuando la variable dependiente es $\lambda \operatorname{lnGDP}$, las variables $I$ son $\operatorname{lnGDP} \_1$, lnPROT, lnINV y lnPOP. Para las regresiones donde la variable dependiente es $\operatorname{lnINV}$, las variables $I$ son lnGDP_1, InPROT y lnPOP, y cuando la variable dependiente es RES no usamos ninguna variable $I$.

Se seleccionaron ocho variables dudosas de la amplia gama de variables usadas en la literatura: crecimiento anual de la relación de intercambio (тот), inflación anual (INF), superávit del gobierno (GOV), deuda externa pública (DEBT), desviación estándar de los índices de reforma (SIGMA), flujos financieros de las organizaciones internacionales (FLOWS), superávit en cuenta corriente (CA) y crecimiento del crédito interno (DOCRE). La mayoría de estas variables suelen usarse en la literatura como indicadores de políticas de corto plazo (GOV y DOCRE), situación externa (CA y DEBT), situación de los mercados internacionales de bienes (TOT), y estabilidad macroeconómica (INF). FLOws se

\footnotetext{
${ }^{14}$ Excluimos el crecimiento del capital humano y el crecimiento de la población puesto que no hay canales claros a través de los cuales las reformas estructurales puedan mejorarlos.
}

incluyó porque los flujos de capital de las organizaciones financieras internacionales tienen un impacto decisivo sobre el desempeño económico de los países en desarrollo. Por último, SIGMA se seleccionó para controlar la heterogeneidad del proceso de reforma.

\section{Los modelos}

Cada modelo incluirá las respectivas variables $I$, la variable central y hasta dos variables dudosas. Por lo tanto, incluidas las variables ficticias, tenemos hasta 10 regresores en las regresiones de $\Delta$ lnGDP, hasta nueve en las regresiones de $\operatorname{lnINV}$, y hasta tres en las regresiones de RES. ${ }^{15} \mathrm{El}$ cuadro 2 muestra la matriz de correlación de las variables utilizadas en el análisis.

Según lo mencionado en la sección 4 , se ha eliminado toda variable dudosa con un coeficiente de correlación mayor que 0.5 con respecto a las variables centrales. Además, DEBT y CA, DEBT y GOVSUR, INF y GOVSUR, DOCRE e INF, y DOCRE y GOVSUR no entran jamás en la misma regresión, puesto que sus coeficientes de correlación respectivos son mayores que 0.5 .

\section{Variables endógenas y exógenas}

Suponemos que todas las variables, con la excepción de тот y las variables centrales, son endógenas con respecto al crecimiento. ${ }^{16}$ Dado que INV y RES son fuentes de crecimiento, consideramos los supuestos de endogeneidad válidos para las regresiones con cualquiera de nuestras tres variables dependientes. Nótese que el crecimiento de la relación de intercambio (тот) es claramente exógeno. Sin embargo, consideramos que los flujos financieros (FLOWS) son endógenos, puesto que la asistencia de las organizaciones financieras internacionales suele llegar en períodos de mal desempeño económico. Por último, todas las variables centrales son consideradas exógenas, bajo el supuesto de que dependen exclusivamente de decisiones de política.

\footnotetext{
${ }^{15}$ Cuando la variable dependiente es REs, las regresiones no incluyen variables ficticias.

${ }^{16}$ Nótese que, tal como se demostró en la sección 3, GDP_1 es endógeno en el modelo en primeras diferencias, puesto que se incluyeron para el cálculo de RES.
} 
CUADRO 2

Matriz de correlación

\begin{tabular}{|c|c|c|c|c|c|c|c|c|c|c|c|c|c|c|c|c|c|c|c|c|c|}
\hline & $\mathrm{d} \operatorname{lnGDP}$ & InINV & RES & InGDP_1 & InPROT & InPOP & $\mathrm{CA}$ & DEBT & FLOWS & INF & TOT & GOVSUR & DOCRE & FIN & $L A B$ & PRIV & TAX & TRADE & SPI & SIGMA & POLCOM \\
\hline $\mathrm{dlnGDP}$ & 1.00 & & & & & & & & & & & & & & & & & & & & \\
\hline InINV & 0.34 & 1.00 & & & & & & & & & & & & & & & & & & & \\
\hline RES & 0.32 & -0.04 & 1.00 & & & & & & & & & & & & & & & & & & \\
\hline InGDP_1 & 0.11 & -0.08 & 0.11 & 1.00 & & & & & & & & & & & & & & & & & \\
\hline InPROT & 0.20 & 0.08 & 0.08 & 0.01 & 1.00 & & & & & & & & & & & & & & & & \\
\hline InPOP & -0.09 & 0.21 & -0.02 & -0.49 & 0.03 & 1.00 & & & & & & & & & & & & & & & \\
\hline $\mathrm{CA}$ & 0.09 & -0.23 & 0.11 & 0.65 & 0.12 & -0.33 & 1.00 & & & & & & & & & & & & & & \\
\hline DEBT & -0.41 & 0.01 & -0.16 & -0.47 & -0.16 & 0.16 & -0.57 & 1.00 & & & & & & & & & & & & & \\
\hline FLOWS & -0.27 & -0.20 & -0.33 & -0.32 & -0.12 & 0.27 & -0.18 & 0.41 & 1.00 & & & & & & & & & & & & \\
\hline INF & -0.46 & -0.09 & -0.02 & 0.04 & -0.10 & 0.00 & -0.22 & 0.40 & -0.04 & 1.00 & & & & & & & & & & & \\
\hline TOT & 0.07 & 0.20 & -0.16 & -0.17 & -0.02 & 0.03 & -0.26 & 0.13 & 0.06 & -0.06 & 1.00 & & & & & & & & & & \\
\hline GOVSUR & 0.45 & 0.24 & -0.03 & 0.17 & 0.20 & -0.13 & 0.34 & -0.48 & -0.17 & -0.69 & 0.22 & 1.00 & & & & & & & & & \\
\hline DOCRE & -0.41 & -0.12 & -0.09 & 0.07 & -0.07 & 0.02 & -0.20 & 0.38 & 0.05 & 0.94 & -0.11 & -0.72 & 1.00 & & & & & & & & \\
\hline FIN & 0.37 & -0.07 & -0.17 & 0.11 & -0.01 & -0.31 & -0.02 & -0.11 & 0.12 & -0.20 & 0.11 & 0.10 & -0.10 & 1.00 & & & & & & & \\
\hline LAB & 0.18 & 0.36 & -0.07 & 0.03 & -0.02 & -0.38 & 0.04 & -0.10 & -0.36 & -0.18 & 0.20 & 0.23 & -0.19 & 0.12 & 1.00 & & & & & & \\
\hline PRIV & 0.24 & 0.18 & -0.06 & 0.18 & -0.02 & -0.17 & -0.06 & 0.07 & 0.09 & -0.14 & 0.11 & 0.13 & -0.11 & 0.25 & 0.03 & 1.00 & & & & & \\
\hline TAX & 0.33 & 0.04 & -0.03 & -0.33 & 0.16 & 0.12 & -0.13 & -0.03 & -0.01 & -0.13 & 0.06 & 0.25 & -0.08 & 0.14 & -0.02 & 0.03 & 1.00 & & & & \\
\hline TRADE & 0.39 & 0.11 & -0.05 & -0.12 & 0.06 & -0.07 & -0.19 & 0.06 & -0.06 & -0.18 & 0.10 & 0.13 & -0.15 & 0.42 & 0.04 & 0.37 & 0.47 & 1.00 & & & \\
\hline SPI & 0.51 & 0.18 & -0.14 & -0.01 & 0.04 & -0.30 & -0.11 & -0.05 & -0.05 & -0.28 & 0.19 & 0.26 & -0.21 & 0.75 & 0.37 & 0.55 & 0.47 & 0.78 & 1.00 & & \\
\hline SIGMA & 0.06 & -0.05 & -0.09 & -0.31 & -0.04 & -0.19 & -0.11 & 0.08 & -0.06 & -0.09 & 0.06 & 0.05 & -0.10 & 0.21 & 0.37 & -0.34 & 0.43 & 0.49 & 0.38 & 1.00 & \\
\hline POLCOM & 0.52 & 0.21 & -0.11 & 0.14 & 0.07 & -0.23 & -0.07 & -0.09 & -0.02 & -0.26 & 0.17 & 0.26 & -0.18 & 0.70 & 0.21 & 0.76 & 0.29 & 0.60 & 0.89 & -0.08 & 1.00 \\
\hline
\end{tabular}

\section{VI}

\section{Resultados econométricos}

\section{El modelo de Solow}

El cuadro 3 muestra la estimación del modelo de Solow. Reportamos aquí las estimaciones de la primera y segunda etapas, pero sólo nos referimos a estos últimos resultados.

Todas las variables explicativas tienen el signo predicho por la teoría, y todas salvo lnPROT son significativas al $95 \%$ de nivel de confianza. Nótese la considerable significación del coeficiente de inversión, que fue la única variable correlacionada robustamente con el crecimiento en el estudio de Levine y Renelt (1992), así como la fuerte significación del coeficiente negativo del PIB per cápita retardado un período, lo que comprueba la existencia de una convergencia condicional. La rutinaria prueba $F$ de significación conjunta rechaza con firmeza la hipótesis nula. Además, el valor crítico para la prueba de Sargan, utilizando un nivel de confianza de $95 \%$, es $\chi_{20}^{2}=31.41$ y por ende no podemos rechazar la hipótesis nula de validez de los instrumentos. Por último, el número de condición de la matriz de variables explicativas no muestra problemas de multicolinealidad.

\section{Matriz de correlación}

La matriz de correlación del cuadro 2 ya ilustró algunos resultados. En ella, todos los índices muestran la correlación prevista con el crecimiento y la inversión, con la excepción de sigma (signo equivocado con respecto al crecimiento) y FIN (signo equivocado con respecto a la inversión). Nótese asimismo la elevada correlación entre crecimiento y SPI antes de controlar las tendencias temporales.

Un resultado sorprendente en esta etapa es la correlación negativa de los índices y la complementariedad de políticas con respecto a RES, nuestra aproximación al crecimiento no observable de la productividad total de los factores. La única variable central cuyo coeficiente de correlación con respecto a RES muestra el signo previsto es SIGMA. Estos resultados son más sorprendentes si se considera que la manera más natural en que las políticas de libre mercado puedan fomentar el crecimiento es mejorando la asignación de recursos. 


\section{Resultados del análisis de límites extremos}

El cuadro 4 muestra los límites extremos de $\uparrow_{F}$ para cada variable central, y el valor de $\uparrow_{F}$ en la respectiva mejor especificación según el criterio de información de Akaike (AIC). El apéndice D describe con mayor detalle los modelos correspondientes y los resultados econométricos.

CUADRO 3

Estimación del modelo de Solow

Variable dependiente: $\lambda$ InGDP

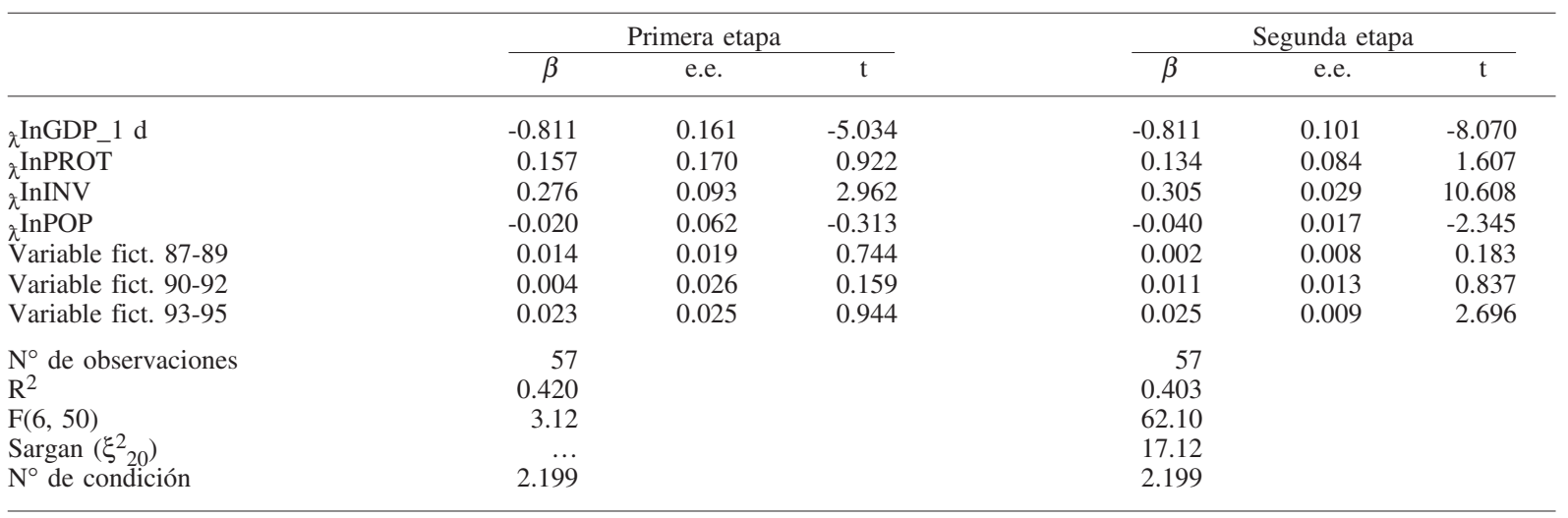

CUADRO 4

Análisis de límites extremos ${ }^{a}$

\begin{tabular}{|c|c|c|c|c|c|c|c|c|c|}
\hline & \multicolumn{3}{|c|}{ Crecimiento } & \multicolumn{3}{|c|}{ Inversión } & \multicolumn{3}{|c|}{ Residuos $^{\mathrm{b}}$} \\
\hline & Alto & Optimo & Bajo & Alto & Optimo & Bajo & Alto & Optimo & Bajo \\
\hline \multirow[t]{2}{*}{ FIN: liberalización financiera } & 0.0306 & -0.0500 & -0.2086 & 0.2771 & -0.0284 & -0.1947 & -0.0351 & $-0.0782^{\mathrm{c}}$ & -0.0763 \\
\hline & 0.0913 & 0.0452 & 0.1068 & 0.1995 & 0.0406 & 0.1784 & 0.0451 & 0.0193 & 0.0396 \\
\hline \multirow[t]{2}{*}{ LAB: flexibilización laboral } & -0.0708 & -0.4487 & -1.0691 & 2.6815 & 0.7962 & -0.5184 & $-0.3752^{\mathrm{c}}$ & $-0.4932^{\mathrm{c}}$ & $-0.5306^{\mathrm{c}}$ \\
\hline & 0.6592 & 0.3642 & 0.6490 & 1.5789 & 0.6296 & 1.2935 & 0.0403 & 0.0266 & 0.0475 \\
\hline \multirow[t]{2}{*}{ PRIV: privatización } & 0.1986 & 0.0559 & -0.2646 & -0.3065 & 0.0309 & -0.4752 & $0.0400^{\mathrm{c}}$ & $-0.1658^{c}$ & $-0.1626^{c}$ \\
\hline & 0.2014 & 0.0522 & 0.1398 & 0.4362 & 0.0641 & 0.4748 & 0.0191 & 0.0060 & 0.0154 \\
\hline \multirow[t]{2}{*}{ TAX: neutralidad tributaria } & 0.1215 & 0.0927 & 0.0110 & 0.2099 & -0.0901 & $-0.3488^{c}$ & $0.0889^{c}$ & $0.0813^{c}$ & $-0.0547^{c}$ \\
\hline & 0.0779 & 0.0493 & 0.1230 & 0.2329 & 0.0953 & 0.1394 & 0.0170 & 0.0162 & 0.0158 \\
\hline \multirow[t]{2}{*}{ TRADE: liberalización comercial } & 0.1441 & -0.0325 & -0.1023 & 0.1791 & 0.1093 & -0.2531 & $0.0641^{\mathrm{c}}$ & $-0.0347^{\mathrm{c}}$ & $0.0078^{\mathrm{c}}$ \\
\hline & 0.1566 & 0.0440 & 0.1418 & 0.3338 & 0.0941 & 0.1950 & 0.0290 & 0.0079 & 0.0160 \\
\hline \multirow{2}{*}{$\begin{array}{l}\text { SPI: promedio de los índices } \\
\text { de políticas }\end{array}$} & 0.2421 & -0.2262 & -0.5974 & $1.0711^{\mathrm{c}}$ & -0.3351 & -0.7132 & -0.1271 & $-0.3256^{\mathrm{c}}$ & $-0.2260^{c}$ \\
\hline & 0.2658 & 0.1942 & 0.4161 & 0.3578 & 0.3398 & 0.5054 & 0.0997 & 0.0552 & 0.1095 \\
\hline \multirow{2}{*}{$\begin{array}{l}\text { SIGMA: error estándar } \\
\text { de los índices de políticas }\end{array}$} & 0.1576 & -0.5455 & -0.7131 & 0.4938 & -0.1212 & $-0.9358^{\mathrm{c}}$ & -0.2389 & $-0.4693^{\mathrm{c}}$ & $-0.5180^{c}$ \\
\hline & 0.4115 & 0.2425 & 0.3666 & 0.5869 & 0.3725 & 0.4037 & 0.1524 & 0.0340 & 0.0836 \\
\hline \multirow{2}{*}{$\begin{array}{l}\text { POLCOM: complementariedad de políticas } \\
\text { (SPI-SIGMA) }\end{array}$} & 0.1713 & 0.0914 & -0.0581 & $0.9284^{\mathrm{c}}$ & 0.1359 & -0.4311 & 0.0326 & -0.0723 & -0.0877 \\
\hline & 0.3123 & 0.2267 & 0.2606 & 0.4628 & 0.0852 & 0.4504 & 0.0849 & 0.0373 & 0.0770 \\
\hline
\end{tabular}

a Las cifras en cursivas corresponden a errores estándares.

Alto $\beta_{F} \quad$ : Coeficiente central correspondiente al máximo límite superior $\left(\beta_{F}+\right.$ dos e.e.)

Optimo $\beta_{F}$ : Coeficiente central correspondiente a la mejor regresión de acuerdo con el criterio de información de Akaike.

Bajo $\beta_{F}$ : Coeficiente central correspondiente al mínimo límite inferior $\left(\beta_{F}\right.$ - dos e.e.)

Con la excepción de SIGMA, el signo previsto de los coeficientes es positivo.

b Residuos del modelo de Solow estimado.

c Coeficiente significativo al $5 \%$ de nivel de significancia. 
El análisis de límites extremos muestra que ninguna variable central se correlaciona robustamente y de la manera teóricamente prevista con el crecimiento o sus fuentes. Esta falta de robustez incluye el promedio de los índices de políticas (SPI) y la complementariedad de políticas (POLCOM=SPI-SIGMA). Nótese que las regresiones con POLCOM representan la versión restringida de los modelos que utilizan SPI y SIGMA, puesto que imponen $\uparrow_{\mathrm{SPI}}=-\uparrow_{\mathrm{SIGMA}}$. Sin embargo, los modelos sin restringir también dan fragilidad. De hecho, el límite mínimo del coeficiente del SPI en las regresiones de crecimiento incluye a SIGMA como variable dudosa (véase el apéndice D).

La única relación robusta es la de LAB con los residuos del modelo de Solow, pero la correlación es negativa, lo que indicaría que la flexibilización laboral está en realidad perjudicando el crecimiento de la productividad. Aunque frágil, la variable central de mejor desempeño es SIGMA, cuyo coeficiente siempre tiene el signo previsto en las regresiones de residuos; esto indicaría que la dispersión de políticas reduce el crecimiento al disminuir la productividad total de los factores.

El cuadro 4 indica también que FIN, LAB, TRADE y SPI en el caso de las regresiones de crecimiento, FIN, TAX y SPI en el caso de las regresiones de inversión, y FIN, PRIV, TRADE y SPI en el caso de las regresiones de residuos, entran en la mejor especificación respectiva con signo negativo. Por tanto, en una búsqueda sistemática y objetiva de la especificación óptima, deberíamos concluir que estas reformas están reduciendo el crecimiento, la inversión y el crecimiento de la productividad, respectivamente.

El cuadro 5 presenta la distribución de $\uparrow_{F}$ conforme al signo y significación. Los resultados muestran que la fragilidad no se debe sólo a unas pocas excepciones. Más bien, FIN, LAB y SPI en las regresiones de crecimiento, y FIN y SPI en las regresiones de residuos, muestran casi siempre una correlación negativa con las

CUADRO 5

Signo y significación de los coeficientes centrales ${ }^{a}$

(Porcentajes)

\begin{tabular}{|c|c|c|c|c|c|c|c|c|c|c|c|c|}
\hline & \multicolumn{4}{|c|}{ Crecimiento } & \multicolumn{4}{|c|}{ Inversión } & \multicolumn{4}{|c|}{ Residuos $^{\mathrm{b}}$} \\
\hline & \multicolumn{2}{|c|}{$\beta_{F}$ positivo } & \multicolumn{2}{|c|}{$\beta_{F}$ negativo } & \multicolumn{2}{|c|}{$\beta_{F}$ positivo } & \multicolumn{2}{|c|}{$\beta_{F}$ negativo } & \multicolumn{2}{|c|}{$\beta_{F}$ positivo } & \multicolumn{2}{|c|}{$\beta_{F}$ negativo } \\
\hline & $\overline{S^{c}}$ & $\mathrm{NS}^{\mathrm{d}}$ & NS & $\bar{S}$ & S & NS & $\overline{\mathrm{NS}}$ & $\bar{S}$ & $\mathrm{~S}$ & $\mathrm{NS}$ & $\overline{\mathrm{NS}}$ & $\mathrm{S}$ \\
\hline $\begin{array}{l}\text { FIN: } \\
\text { Liberalización financiera }\end{array}$ & 0 & 14 & 75 & 9 & 28 & 59 & 9 & 3 & 0 & 0 & 38 & 63 \\
\hline $\begin{array}{l}\text { LAB: } \\
\text { Flexibilización laboral }\end{array}$ & 0 & 0 & 44 & 5 & 28 & 63 & 9 & 0 & 0 & 0 & 0 & 100 \\
\hline $\begin{array}{l}\text { PRIV: } \\
\text { Privatización }\end{array}$ & 13 & 66 & 16 & 6 & 0 & 59 & 4 & 0 & 13 & 38 & 2 & 28 \\
\hline $\begin{array}{l}\text { TAX: } \\
\text { Neutralidad tributaria }\end{array}$ & 3 & 66 & 31 & 0 & 0 & 31 & 4 & 25 & 22 & 6 & 31 & 41 \\
\hline $\begin{array}{l}\text { TRADE: } \\
\text { Liberalización comercial }\end{array}$ & 0 & 53 & 47 & 0 & 3 & 59 & 38 & 0 & 19 & 6 & 33 & 38 \\
\hline $\begin{array}{l}\text { SPI: } \\
\text { Promedio de los índices de } \\
\text { políticas }\end{array}$ & 0 & 19 & 72 & 9 & 9 & 56 & 34 & 0 & 0 & 0 & 16 & 84 \\
\hline $\begin{array}{l}\text { SIGMA: } \\
\text { Error estándar de los } \\
\text { índices de políticas }\end{array}$ & 0 & 13 & 75 & 13 & 4 & 50 & 33 & 13 & 0 & 0 & 4 & 96 \\
\hline POLCOM: SPI-SIGMA & 0 & 54 & 46 & 0 & 8 & 54 & 38 & 0 & 4 & 33 & 46 & 17 \\
\hline
\end{tabular}

a Los coeficientes centrales son significativos o no significativos al 5\% de nivel de significación. Número de modelos: FIN, LAB, PRIV, TAX, TRADE y SPI: 32 modelos. SIGMA y POLCOM: 24 modelos.

b Residuos del modelo de Solow.

c $\mathrm{S}=$ Significativo.

d NS= No significativo. 
variables dependientes. Con respecto a la inversión, las variables centrales son generalmente insignificantes.

\section{4. ¿Mala suerte o malas políticas?}

Puesto que estamos controlando los efectos temporales, los resultados del análisis de límites extremos arrojan serias dudas sobre la eficacia de las reformas de políticas, sean los factores internacionales positivos o negativos. No obstante lo anterior, Easterly, Loayza y Montiel (1997), representando la opinión

\section{VII \\ Conclusiones}

Este artículo ha sostenido implícitamente que el control de efectos fijos, endogeneidad, correlación espuria e incertidumbre del modelo, así como el establecimiento de procedimientos objetivos en la selección de modelos para evitar los sesgos del investigador, deben ser procedimientos estándares en las regresiones de crecimiento, sobre todo cuando los resultados tienen grandes implicaciones de política.

Considerando todas estas premisas, el estudio muestra que la evidencia del impacto positivo de las reformas estructurales sobre el crecimiento latinoamericano dista de ser concluyente. De hecho, nuestro análisis arroja serias dudas sobre la eficacia de las reformas, puesto que ninguna se correlaciona robustamente y con el signo previsto con el crecimiento o sus fuentes; las reformas frecuentemente entran con signo negativo en las respectivas mejores especificaciones del crecimiento y sus fuentes, y no es difícil hallar especificaciones en que los índices de reformas tienen coeficientes negativos y significativos.

Es más, la única relación robusta encontrada en nuestro análisis es una correlación negativa entre la flexibilización laboral (LAB) y los residuos del modelo de Solow (RES), lo que indicaría que la flexibilización laboral está perjudicando la productividad. La liberalización financiera (FIN) y el promedio de los índices de políticas (SPI) también muestran sólo coeficientes negativos en las regresiones RES, lo que indicaría que estarían perjudicando la productividad. Dado que las reformas del Consenso de Washington deberían fomentar el crecimiento, sobre todo a través de la mejora en la asignación de recursos, estos resultados sugieren serias dudas en cuanto a la eficacia de las reformas en América Latina. general sobre el tema, argumentaron que el mal desempeño económico de América Latina, a pesar de las reformas, se debía a un entorno económico internacional adverso.

Sin embargo, la estimación del modelo de Solow en el cuadro 3 presenta variables ficticias positivas, lo que indica que el período en consideración, si de algo puede ser calificado, es de favorable en lo internacional para América Latina. Este resultado es compatible con el comportamiento de la tasa de interés internacional, como se destacó en la sección III. ${ }^{17}$
La falta de robustez de la correlación positiva entre reformas y crecimiento no mejora al controlar por la dispersión de las políticas (SIGMA), ya sea utilizando un índice de complementariedad de políticas (POLCOM=SPI-SIGMA) o regresiones sin restricciones. Sin embargo, en general el coeficiente de SIGMA muestra el signo previsto cuando entra en las regresiones con RES, lo que indicaría que la disparidad en el nivel de reformas afecta a la productividad.

Por último, aunque las conclusiones precedentes no se vean afectadas por la dirección de los efectos temporales durante el período, estos efectos, si algo, han sido positivos, lo que indicaría que el mal desempeño económico de América Latina no se debió a un entorno internacional adverso. Este resultado es consistente con el comportamiento de la tasa de interés internacional durante el período, la cual es una variable decisiva para el crecimiento regional.

Este estudio ha utilizado los mismos índices de reformas que aquéllos usados por la mayor parte de la evidencia cuestionada, y, aunque perfectibles, dichos índices representaron una importante mejora para medir las reformas estructurales latinoamericanas,

\footnotetext{
${ }^{17}$ Como se mencionó en la sección III, la especificación de efectos fijos impide identificar los coeficientes de cualquier variable temporal o individualmente invariante. Por lo tanto, técnicamente no podemos descartar que "los buenos tiempos" se hayan producido por un SPI regional más elevado por período. Sin embargo, dados los resultados del análisis de límites extremos, es improbable que mientras los cambios en el SPI por países no están correlacionados robustamente con el crecimiento, los cambios del promedio regional sí lo estén.
} 
puesto que incorporaron explícitamente variables de política y no supuestos resultados de dichas reformas.

Finalmente, en el futuro la disponibilidad de series temporales más largas y tal vez mejores índices pueden echar más luz sobre el verdadero impacto de las reformas de mercado en el crecimiento latinoamericano. Sin embargo, de verificarse las conclusiones del presente estudio, sería prioritario investigar cómo políticas destinadas a mejorar la asignación de recursos de hecho pueden perjudicar la productividad. En esta tarea, los estudios de casos podrían ser más esclarecedores que las regresiones econométricas.

Mientras tanto ¿puede alguien sugerir que se aceleren las reformas?

APÉNDICE A

\section{Medición de las reformas estructurales}

Los componentes de los índices de políticas son:

Liberalización financiera:

a) Libertad de tasas de interés de depósitos

b) Libertad de tasas de interés de los créditos

c) Nivel efectivo de encaje de los depósitos bancarios

d) Calidad de la supervisión bancaria y financiera

Flexibilización laboral:

a) Flexibilidad de contratación

b) Costos de despido al año de trabajo

c) Costos de despido a los 10 años de trabajo

d) Sobrecostos por jornadas extraordinarias

e) Contribuciones a la seguridad social

Privatización:

Montos acumulados por privatizaciones desde 1988

Neutralidad tributaria:

a) Tasa marginal máxima de tributación sobre el ingreso de las sociedades

b) Tasa marginal máxima de tributación sobre el ingreso de las personas c) Tasa básica del impuesto al valor agregado

d) Productividad del impuesto al valor agregado ${ }^{18}$

Liberalización comercial:

a) Aranceles promedios

b) Dispersión arancelaria

Si cuantificamos cada observación de la variable de política respectiva de modo que los valores más elevados representen más neutralidad, el valor de cada índice $R$ del país $j$ en el año $t$ está dado por la función

$$
\boldsymbol{R}_{t}^{j}=\frac{1}{n} \sum_{i=1}^{n} \frac{x_{i t}^{j}-x_{i}^{\min }}{x_{i}^{\max }-x_{i}^{\min }}
$$

donde $n$ es el número de variables de políticas incluidas en el índice $R, x_{i t}^{j}$ es la observación de la variable $i$ para el país $j$ en el año $t$, y $x_{i}^{\min }\left(x_{i}^{\max }\right)$ es el valor mínimo (máximo) observado de la variable $i$ en todos los países y años.
APENDICE B

\section{Aspectos econométricos}

a) Estimación consistente de $\uparrow$

Suponiendo por simplicidad notacional paneles equilibrados, los modelos que van a estimarse tienen la forma general:

$$
\begin{aligned}
y_{i t} & =\mathrm{x}_{\mathrm{it}}{ }^{\prime} \mathfrak{\downarrow}+\square_{\mathrm{t}}+\mapsto i+v_{i t} \\
\mathrm{i} & =1,2, \ldots, \mathrm{N} . \mathrm{t}=2, \ldots, \mathrm{T}
\end{aligned}
$$

donde $\mathrm{x}_{\text {it }}$ es el vector de variables explicativas $K$ x 1 que puede contener la variable dependiente retardada en un período, regresores endógenos $\left(\mathrm{E}\left[\mathrm{x}^{1}{ }_{\text {it }} v_{\text {is }}\right] \gamma 0\right.$ para $\left.\mathrm{t}=\mathrm{s}\right)$, y regresores estrictamente exógenos $\left(\mathrm{E}^{\mathrm{x}^{2}}{ }_{\text {it }} v_{i s}\right]=0$ para todos los $\left.t, s\right) ; \uparrow$ es el vector de constantes $K$ x $1 ; \square, \mathrm{y} \mapsto$, son los efectos temporales e individuales no observables, respectivamente; $v_{i t}$ es el término de error; $\mathrm{y}$ en general $E\left[\square_{i} \mid \mathrm{x}_{\mathrm{it}}\right] \gamma 0$.

Tomando primeras diferencias para barrer los efectos individuales, e introduciendo variables ficticias para estimar los efectos temporales, tenemos

$$
\begin{gathered}
\lambda y_{i t}=\lambda \mathrm{x}_{\mathrm{it}}{ }^{\prime} \uparrow+\mathrm{d}_{\mathrm{t}}{ }^{\prime} \phi+\lambda v_{i t} \\
\mathrm{i}=1,2, \ldots, \mathrm{N} . \mathrm{t}=2, \ldots, \mathrm{T} .
\end{gathered}
$$

donde $d_{t}$ es un vector de variables ficticias $(T-2)$ x 1 representando los períodos $3,4, \ldots$, у $\phi$ es un vector de constantes $(T-2) \times 1$. Si tenemos $y_{\mathrm{it}-1}$ en el lado derecho de [B.1], la estimación мCo de $\uparrow$ en
[B.2] es inconsistente. En este caso, tenemos endogeneidad de regresores puesto que $E\left[\Delta y_{i t-1} \Delta v_{i t}\right] \gamma 0$ debido al hecho de que $y_{\mathrm{it}-1} \mathrm{y}$ $v_{i t-I}$ están correlacionados. Un problema similar se presenta si consideramos algunas variables $x_{\mathrm{it}}$ como endógenas. Sin embargo, si suponemos que $v_{i t}$ no está correlacionada serialmente y que los regresores endógenos también son débilmente exógenos en el sentido de que $E\left[x_{i t}^{I} v_{i s}\right]=0$ para $t<s$, cualquier valor de $y_{i t}$ o de $x^{I}{ }_{i t}$ retardado en dos o más períodos son instrumentos válidos para [B.2], puesto que $E\left[y_{i t-2} \lambda v_{i t}\right]=0$ y $E\left[\mathrm{x}^{1}{ }_{\mathrm{it}-2} \lambda v_{i t}\right]=0$. Por ende, bajo los supuestos de identificación precedentes y teniendo $T \mu 3$ existen instrumentos para hacer una estimación consistente de $\uparrow$.

b) El estimador Gмм en dos etapas

El estimador GMM en dos etapas propuesto por Arellano y Bond (1991) usa la matriz instrumental $W^{A B}=\left(Z^{A B}, d\right)$, donde $d$ es la matriz de variables ficticias, $Z^{A B}=\left(Z^{A B}{ }_{1}^{\prime} \ldots . . Z^{A B}{ }_{N}{ }^{\prime}\right)^{\prime}, \mathrm{y} Z^{A B}{ }_{i}=\operatorname{diag}\left(y_{i l}, \ldots, y_{i t-2} ;\right.$ $\left.x_{i 1}^{l}, \ldots, x_{i t-2}^{l}, ; x_{i 1}^{2}, \ldots, x^{2}{ }_{i T}{ }^{\prime}\right)$. Esta matriz instrumental aprovecha óptimamente la ortogonalidad con respecto a $\lambda v_{i t}$ de los valores de $y_{i t}$ y $x_{i t}^{1}$ retardados en dos o más períodos y de todos los valores de

${ }^{18}$ Definido como la relación entre la tasa básica y la recaudación expresada en porcentaje del PIB. 
$x^{2}{ }_{i t}$. En la estimación real usamos un subconjunto de las condiciones de ortogonalidad precedentes, eligiendo la matriz instrumental $W=(Z, d)$, donde $Z=\left(z_{l}{ }^{\prime}, \ldots, z_{N}{ }^{\prime}\right), y z_{i}=\operatorname{diag}\left(y_{i l} ; \ldots, y_{i t-2} ; x_{i l}^{l}{ }_{i l}\right.$, $\left.\ldots, x^{I}{ }_{i t-2}, ; \Delta x^{2}{ }_{i 3}, \ldots, \Delta x^{2}{ }_{i t}\right)$.'.

La estimación se hace en dos etapas. Primero, se realiza la estimación de [B.2] con variables instrumentales usando la matriz $W$. Esta estimación es consistente, pero ineficiente si $\lambda v_{i t}$ no es homocedástica. Con los residuos de esta primera estimación se estima una matriz de covarianza simple para $\lambda v_{i t}$ y se realiza una estimación de [B.2], usando nuevamente la matriz $W$ y la matriz de covarianza anteriormente estimada.

Definiendo $\Delta \tilde{X}=[\Delta X, d]$, el estimador gмm en dos etapas tiene la forma:

$$
\hat{\beta}=\left[\left(\Delta \tilde{\mathrm{X}}^{\prime} \mathrm{W}\right) \mathrm{A}_{\mathrm{N}}\left(\mathrm{W}^{\prime} \Delta \tilde{\mathrm{X}}\right)\right]^{-1}\left[\left(\Delta \tilde{\mathrm{X}}^{\prime} \mathrm{W}\right) \mathrm{A}_{\mathrm{N}}\left(\mathrm{W}^{\prime} \Delta \mathrm{Y}\right)\right]
$$

donde $A_{N}=\left[\sum_{i} W_{i}^{\prime}\left(\Delta \hat{v}_{i}\right)\left(\Delta \hat{v}_{i}\right)^{\prime} W_{i}\right]^{-1}$ y donde $\Delta \hat{v}_{i}$ es el vector de residuos de la estimación de la primera etapa, es decir, omitiendo $\mathrm{A}_{\mathrm{N}}$ en [B.3].

c) Test de Sargan de restricciones de sobreidentificación

Dado que tenemos más instrumentos que parámetros por estimar, los modelos están sobreidentificados. Con estas restricciones de sobreidentificación podemos verificar la validez de los instrumentos mediante el estadístico

$$
s=\Delta \breve{v}^{\prime} W\left[\sum_{i} W_{i}^{\prime}\left(\Delta \breve{v}_{i}\right)\left(\Delta \breve{v}_{i}\right)^{\prime} W_{i}\right]^{-1} W^{\prime} \Delta \breve{v}
$$

donde $\Delta \breve{v}$ es el vector de residuos de la segunda etapa. Bajo la hipótesis nula de validez de los instrumentos, el estadístico $s$ se distribuye asintóticamente como $\chi_{r}^{2}$, donde $r$ es el número de restricciones de sobreidentificación, o sea, la diferencia entre las columnas de $W$ y el número de coeficientes estimados. El rechazo de la hipótesis nula sugiere, por ejemplo, que uno de nuestros supuestos -residuos no autocorrelacionados en [B.1] y/o regresores endógenos pero débilmente exógenos- no es verdadero.

APENDICE C

\section{Variables y fuentes}

a) Índices de políticas

FIN : índice de liberalización financiera. Fuente: BID

LAB : índice de flexibilización laboral. Fuente: BID.

PRIV : índice de privatización. Fuente: BID.

TAX : índice de neutralidad tributaria. Fuente: BID.

TRADE : índice de liberalización comercial. Fuente: BID.

SPI : índice de política estructural, definido como la media aritmética de los cinco índices de políticas. Fuente: BID.

SIGMA : dispersión de los índices, definida como la desviación estándar de los cinco índices de políticas.

POLCOM : complementariedad de políticas, definida como SPI-SIGMA.

b) Otras variables

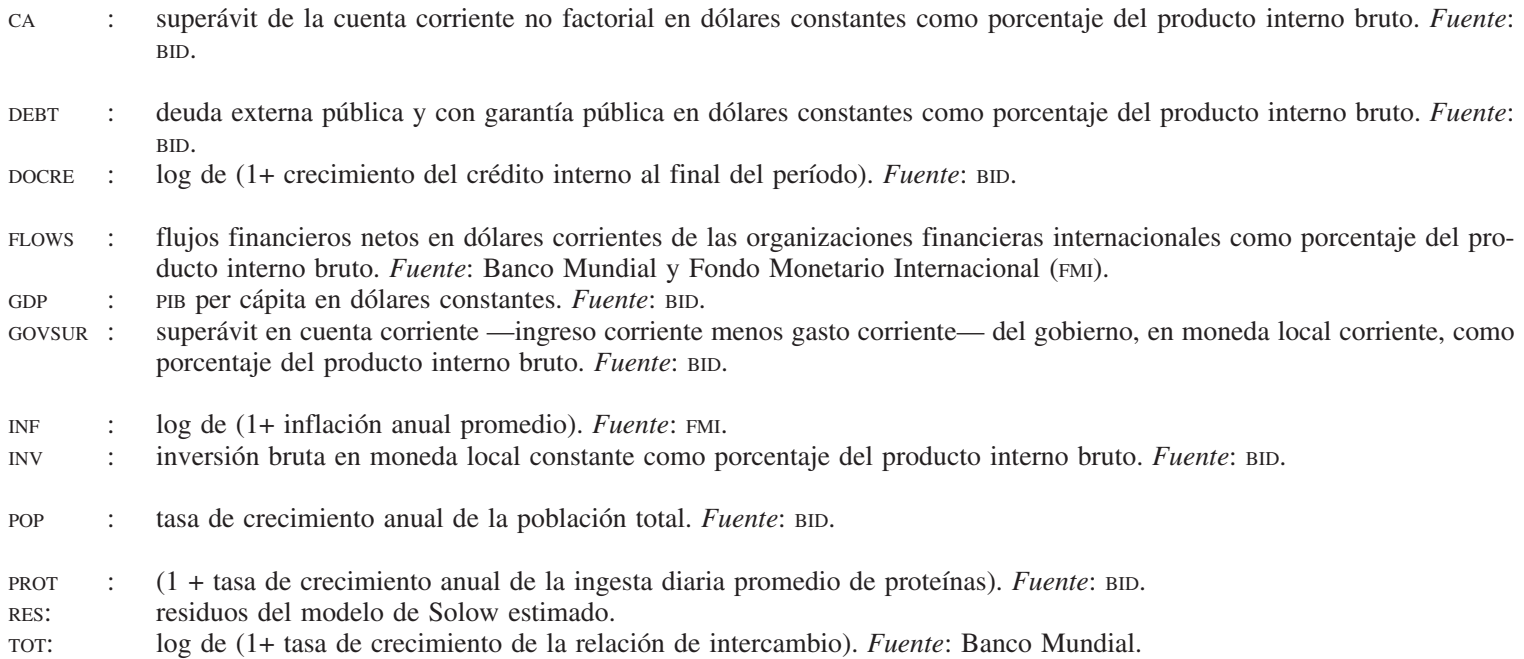


APENDICE D

\section{Análisis de límites extremos ${ }^{a}$}

Variable dependiente : $\Delta \operatorname{lnGDP}$

Variables $I: \quad: \operatorname{lnGDP} \_1, \operatorname{lnPROT}, \operatorname{lnINV}$ y $\operatorname{lnPOP}$

\begin{tabular}{|c|c|c|c|c|c|c|c|c|c|c|}
\hline Variable central & & B & $e^{b}$ & $\mathrm{t}$ & $\mathrm{R}^{2}$ & $\mathrm{AIC}^{\mathrm{c}}$ & Variables $D$ & $\mathrm{Obs}^{\mathrm{d}}$ & Sargan & $\mathrm{N}^{\mathrm{o}}$ de cond. \\
\hline FIN: & alto: & 0.0306 & 0.0913 & 0.3348 & 0.4571 & -5.4092 & DEBT, FLOWS & 57 & \multirow{3}{*}{$\begin{array}{l}\xi^{2}{ }^{35}=28.61 \\
\xi^{2}{ }^{35}=9.35 \\
\xi^{2}{ }_{35}=24.18\end{array}$} & 3.95 \\
\hline Liberalización & óptimo: & -0.0500 & 0.0452 & -1.1053 & 0.5188 & -5.5297 & DEBT, DOCRE & 57 & & 4.64 \\
\hline financiera & bajo: & -0.2086 & 0.1068 & -1.9538 & 0.3245 & -5.1906 & TOT, DOCRE & 57 & & 3.44 \\
\hline LAB: & alto: & -0.0708 & 0.6592 & -0.1074 & 0.3708 & -5.2616 & FLOWS, DOCRE & 57 & \multirow{3}{*}{$\begin{array}{l}\xi^{2}{ }_{35}=32.2 \\
\xi^{2}{ }^{35}=10.58 \\
\xi^{2}{ }_{35}=28.53\end{array}$} & 3.76 \\
\hline Flexibilización & óptimo & -0.4487 & 0.3642 & -1.2320 & 0.5487 & -5.5940 & DEBT, DOCRE & 57 & & 3.95 \\
\hline laboral & bajo: & -1.0691 & 0.6490 & -1.6473 & 0.4347 & -5.3687 & DEBT, FLOWS & 57 & & 3.45 \\
\hline PRIV: & alto: & 0.1986 & 0.2014 & 0.9861 & 0.3351 & -5.2069 & DEBT, SIGMA & 52 & \multirow{3}{*}{$\begin{array}{l}\xi^{2}{ }^{35}=7.17 \\
\xi^{2}{ }^{35}=7.85 \\
\xi^{2}{ }_{35}=20.28\end{array}$} & 5.87 \\
\hline Privatización & óptimo: & 0.0559 & 0.0522 & 1.0724 & 0.4901 & -5.4719 & DEBT, FLOWS & 57 & & 4.07 \\
\hline & bajo: & -0.2646 & 0.1398 & -1.8921 & 0.4069 & -5.3212 & SIGMA, GOVSUR & 52 & & 4.46 \\
\hline TAX: & alto: & 0.1215 & 0.0779 & 1.5609 & 0.3689 & -5.2585 & FLOWS, DOCRE & 57 & \multirow{3}{*}{$\begin{array}{l}\xi^{2} 35=2.83 \\
\xi^{23}=4.14 \\
\xi_{35}^{235}=8.04\end{array}$} & 4.02 \\
\hline Neutralidad & óptimo: & 0.0927 & 0.0493 & 1.8806 & 0.5013 & -5.4940 & DEBT, TOT & 57 & & 7.02 \\
\hline tributaria & bajo: & 0.0110 & 0.1230 & 0.0891 & 0.4187 & -5.3413 & SIGMA, GOVSUR & 52 & & 4.88 \\
\hline TRADE: & alto: & 0.1441 & 0.1566 & 0.9200 & 0.3798 & -5.2765 & DEBT, INF & 52 & \multirow{3}{*}{$\begin{array}{l}\xi^{2}{ }_{35}=20.14 \\
\xi^{25}=18.33 \\
\xi_{35}^{20}=24.79\end{array}$} & 5.39 \\
\hline Liberalización & óptimo: & -0.0325 & 0.0440 & -0.7389 & 0.4525 & -5.4396 & DOCRE & 52 & & 4.61 \\
\hline comercial & bajo: & -0.1023 & 0.1418 & -0.7214 & 0.4622 & -5.4190 & DEBT, DOCRE & 52 & & 5.48 \\
\hline SPI: & alto: & 0.2421 & 0.2658 & 0.9110 & 0.2300 & -5.0986 & INF & 52 & \multirow{3}{*}{$\begin{array}{l}\xi^{2}{ }_{30}=34.41 \\
\xi^{2}{ }_{35}=17.54 \\
\xi^{2}{ }_{35}=14.38\end{array}$} & 6.41 \\
\hline Promedio índices & óptimo: & -0.2262 & 0.1942 & -1.1645 & 0.4687 & -5.4313 & FLOWS, DOCRE & 52 & & 7.19 \\
\hline $\begin{array}{l}\text { sect. de políticas } \\
\text { estructurales }\end{array}$ & bajo: & -0.5974 & 0.4161 & -1.4357 & 0.3326 & -5.2031 & SIGMA, GOVSUR & 52 & & 6.66 \\
\hline SIGMA: & alto: & 0.1025 & 0.4386 & 0.2318 & 0.4344 & -5.3686 & DEBT, TOT & 52 & \multirow{3}{*}{$\begin{array}{l}\xi_{35}^{2}=27.10 \\
\xi_{30}^{35}=24.37 \\
\ldots\end{array}$} & 3.65 \\
\hline Desviación estándar & óptimo: & -0.5455 & 0.2425 & -2.2493 & 0.4441 & -5.4244 & DOCRE & 52 & & 3.70 \\
\hline $\begin{array}{l}\text { de los índices de } \\
\text { políticas estructurales }\end{array}$ & bajo: & -0.7131 & 0.3666 & -1.9448 & 0.3052 & -5.1629 & TOT & 52 & & 2.32 \\
\hline POLCOM: & alto: & 0.1713 & 0.3123 & 0.5484 & 0.3518 & -5.2709 & GOVSUR & 52 & \multirow{3}{*}{$\begin{array}{l}\xi^{2}{ }_{30}=32.28 \\
\xi^{2}{ }_{35}=25.15 \\
\xi_{35}^{2}=26.28\end{array}$} & 4.47 \\
\hline \multirow[t]{2}{*}{ SPI-SIGMA } & óptimo: & 0.0914 & 0.2267 & 0.4029 & 0.4615 & -5.4180 & DEBT, DOCRE & 52 & & 6.55 \\
\hline & bajo: & -0.0581 & 0.2606 & -0.2231 & 0.3386 & -5.2121 & TOT, GOVSUR & 52 & & 6.19 \\
\hline
\end{tabular}

a Alto $\beta_{F}$ : coeficiente central de la regresión con el límite máximo $\left(\beta_{F}=\right.$ dos e.e.).

Óptimo $\beta_{F}$ : coeficiente central de la regresión con el valor mínimo del criterio de información de Akaike (AIC).

Bajo $\beta_{F}$ : coeficiente central de la regresión con el límite mínimo $\beta_{F}$ - dos e.e.)

b ee: error estándar.

c AIC: Criterio de información de Akaike.

d Número de observaciones. 
APENDICE D (Continuación)

Variable dependiente : $\ln I N \mathrm{~N}$

Variables $I \quad: \operatorname{lnGDP} \_1, \ln P R O T$ y $\operatorname{lnPOP}$.

\begin{tabular}{|c|c|c|c|c|c|c|c|c|c|c|}
\hline Variable central & & B & $e e^{b}$ & $\mathrm{t}$ & $\mathrm{R}^{2}$ & $\mathrm{AIC}^{\mathrm{c}}$ & Variables $D$ & $\mathrm{Obs}^{\mathrm{d}}$ & Sargan & $\mathrm{N}^{\mathrm{o}}$ de cond. \\
\hline FIN: & alto: & 0.2771 & 0.1995 & 1.3886 & 0.1092 & -3.4901 & TOT, DOCRE & 57 & $\xi_{30}^{2}=13.80$ & 3.03 \\
\hline Liberalización & óptimo: & -0.0284 & 0.0406 & -0.7000 & 0.6151 & -4.3293 & CA, DOCRE & 57 & $\xi_{30}^{20}=14.69$ & 2.82 \\
\hline financiera & bajo: & -0.1947 & 0.1784 & -1.0914 & 0.2426 & -3.6523 & FLOWS, INF & 57 & $\xi_{30}^{20}=18.42$ & 3.33 \\
\hline LAB: & alto: & 2.6815 & 1.5789 & 1.6983 & -0.1168 & -3.2640 & FLOWS, DOCRE & 57 & $\xi_{30}^{2}=16.38$ & 2.50 \\
\hline Flexibilización & óptimo & 0.7962 & 0.6296 & 1.2646 & 0.6385 & -4.3921 & CA, DOCRE & 57 & $\xi_{30}^{20}=9.81$ & 2.24 \\
\hline laboral & bajo: & -0.5184 & 1.2935 & -0.4008 & -0.1063 & -3.4774 & SIGMA, GOVSUR & 52 & $\xi_{30}^{20}=14.72$ & 2.41 \\
\hline PRIV: & alto: & -0.3065 & 0.4362 & -0.7027 & 0.0289 & -3.6078 & SIGMA, GOVSUR & 52 & $\xi_{30}^{2}=13.12$ & 4.05 \\
\hline \multirow[t]{2}{*}{ Privatización } & óptimo: & 0.0309 & 0.0641 & 0.4818 & 0.6577 & -4.4466 & CA, DOCRE & 57 & $\xi_{30}^{20}=25.31$ & 3.18 \\
\hline & bajo: & -0.4752 & 0.4748 & -1.0007 & 0.0142 & -3.5927 & DEBT, SIGMA & 52 & $\xi_{30}^{20}=13.97$ & 5.09 \\
\hline TAX: & alto: & 0.2099 & 0.2329 & 0.9012 & 0.2673 & -3.6855 & DEBT, FLOWS & 57 & $\xi_{30}^{2}=23.24$ & 6.01 \\
\hline \multirow[t]{2}{*}{ Neutralidad tributaria } & óptimo: & -0.0901 & 0.0953 & -0.9455 & 0.6671 & -4.4743 & CA, DOCRE & 57 & $\xi^{2}{ }_{30}^{30}=28.66$ & 2.43 \\
\hline & bajo: & -0.3488 & 0.1394 & -2.5014 & -0.0101 & -3.5684 & SIGMA, GOVSUR & 52 & $\xi_{30}^{20}=3.97$ & 4.75 \\
\hline TRADE: & alto: & 0.1791 & 0.3338 & 0.5367 & 0.1154 & -3.7011 & DEBT, SIGMA & 52 & $\xi_{30}^{2}=13.29$ & 7.94 \\
\hline \multirow{2}{*}{$\begin{array}{l}\text { Liberalización } \\
\text { comercial }\end{array}$} & óptimo: & 0.1093 & 0.0941 & 1.1616 & 0.5181 & -4.3084 & CA, DOCRE & 52 & $\xi_{30}^{20}=26.58$ & 3.65 \\
\hline & bajo: & -0.2531 & 0.1950 & -1.2980 & 0.0279 & -3.6068 & FLOWS, SIGMA & 52 & $\xi_{30}^{20}=10.21$ & 6.71 \\
\hline SPI: & alto: & 1.0711 & 0.3578 & 2.9937 & 0.0295 & -3.6468 & DEBT & 52 & $\xi_{25}^{2}=17.31$ & 4.12 \\
\hline \multirow{2}{*}{$\begin{array}{l}\text { Promedio índices } \\
\text { sect. de políticas } \\
\text { estructurales }\end{array}$} & óptimo: & -0.3351 & 0.3398 & -0.9859 & 0.5155 & -4.3031 & CA, DOCRE & 52 & $\xi_{30}^{25}=11.74$ & 5.27 \\
\hline & bajo: & -0.7132 & 0.5054 & -1.4112 & 0.4070 & -4.1010 & CA, GOVSUR & 52 & $\xi_{30}^{20}=12.30$ & 5.19 \\
\hline SIGMA: & alto: & 0.4938 & 0.5869 & 0.8412 & 0.1126 & -3.6980 & FLOWS, INF & 52 & $\xi_{30}^{2}=21.26$ & 4.64 \\
\hline \multirow{2}{*}{$\begin{array}{l}\text { Desviación estándar } \\
\text { de los índices de } \\
\text { políticas estructurales }\end{array}$} & óptimo: & -0.1212 & 0.3725 & -0.3255 & 0.5566 & -4.3918 & CA, DOCRE & 52 & $\xi 2_{30}^{30}=20.71$ & 3.51 \\
\hline & bajo: & -0.9358 & 0.4037 & -2.3178 & 0.4888 & -4.2495 & CA, TOT & 52 & $\xi_{30}^{20}=15.63$ & 5.33 \\
\hline POLCOM: & alto: & 0.9284 & 0.4628 & 2.0010 & 0.3259 & -3.9728 & CA, GOVSUR & 52 & $\varepsilon_{30}^{2}=13.71$ & 4.40 \\
\hline \multirow[t]{2}{*}{ SPI-SIGMA } & óptimo: & 0.1359 & 0.0852 & 1.5956 & 0.4994 & -4.2700 & CA, FLOWS & 52 & $\varepsilon^{2}{ }_{30}^{30}=14.61$ & 5.31 \\
\hline & bajo: & -0.4311 & 0.4504 & -0.9572 & -0.0456 & -3.5339 & DEBT, DOCRE & 52 & $\varepsilon_{30}^{20}=23.11$ & 5.98 \\
\hline
\end{tabular}


APENDICE D (Conclusión)

Variable dependiente: RES

Variables $I$ : ninguna

\begin{tabular}{|c|c|c|c|c|c|c|c|c|c|c|}
\hline Variable central & & B & $e e^{b}$ & $\mathrm{t}$ & $\mathrm{R}^{2}$ & $\mathrm{AIC}^{\mathrm{c}}$ & Variables $D$ & $\mathrm{Obs}^{\mathrm{d}}$ & Sargan & $\mathrm{N}^{\mathrm{o}}$ de cond. \\
\hline $\begin{array}{l}\text { FIN: } \\
\text { Liberalización } \\
\text { financiera }\end{array}$ & $\begin{array}{l}\text { alto: } \\
\text { óptimo: } \\
\text { bajo: }\end{array}$ & $\begin{array}{r}0.0351 \\
-0.0783 \\
-0.0763\end{array}$ & $\begin{array}{l}0.0451 \\
0.0193 \\
0.0396\end{array}$ & $\begin{array}{l}-0.7778 \\
-4.0594 \\
-1.9274\end{array}$ & $\begin{array}{l}0.1268 \\
0.1835 \\
0.0743\end{array}$ & $\begin{array}{l}-5.6955 \\
-5.7275 \\
-5.6371\end{array}$ & $\begin{array}{l}\text { DEBT } \\
\text { DEBT, DOCRE } \\
\text { DOCRE }\end{array}$ & $\begin{array}{l}57 \\
57 \\
57\end{array}$ & $\begin{array}{l}\xi^{2}{ }_{10}=11.44 \\
\xi^{2}{ }_{15}=17.92 \\
\xi_{10}^{2}=10.50\end{array}$ & $\begin{array}{l}1.74 \\
2.89 \\
1.74\end{array}$ \\
\hline $\begin{array}{l}\text { LAB: } \\
\text { Flexibilización } \\
\text { laboral }\end{array}$ & $\begin{array}{l}\text { alto: } \\
\text { óptimo } \\
\text { bajo: }\end{array}$ & $\begin{array}{l}-0.3752 \\
-0.4932 \\
-0.5306\end{array}$ & $\begin{array}{l}0.0403 \\
0.0266 \\
0.0475\end{array}$ & $\begin{array}{r}-9.3072 \\
-18.5364 \\
-11.1821\end{array}$ & $\begin{array}{l}0.1657 \\
0.2014 \\
0.1718\end{array}$ & $\begin{array}{l}-5.6360 \\
-5.7498 \\
-5.6433\end{array}$ & $\begin{array}{l}\text { TOT, SIGMA } \\
\text { DEBT, DOCRE } \\
\text { DEBT, SIGMA }\end{array}$ & $\begin{array}{l}52 \\
57 \\
52\end{array}$ & $\begin{array}{l}\xi_{2}^{2}=17.45 \\
\xi_{15}^{2}=17.94\end{array}$ & $\begin{array}{l}1.62 \\
2.47 \\
2.82\end{array}$ \\
\hline $\begin{array}{l}\text { PRIV: } \\
\text { Privatización }\end{array}$ & $\begin{array}{l}\text { alto: } \\
\text { óptimo: } \\
\text { bajo: }\end{array}$ & $\begin{array}{r}0.0400 \\
-0.1658 \\
-0.1627\end{array}$ & $\begin{array}{l}0.0191 \\
0.0060 \\
0.0154\end{array}$ & $\begin{array}{r}2.1005 \\
-27.8000 \\
-10.5302\end{array}$ & $\begin{array}{r}-0.1432 \\
0.1853 \\
0.0558\end{array}$ & $\begin{array}{l}-5.4261 \\
-5.6598 \\
-5.5123\end{array}$ & $\begin{array}{l}\text { DOCRE } \\
\text { TOT, SIGMA } \\
\text { CA, SIGMA }\end{array}$ & $\begin{array}{l}57 \\
52 \\
52\end{array}$ & $\begin{array}{l}\xi_{10}^{2}=10.53 \\
\ldots \\
\xi_{15}^{2}=18.43\end{array}$ & $\begin{array}{l}2.65 \\
2.83 \\
1.53\end{array}$ \\
\hline $\begin{array}{l}\text { TAX: } \\
\text { Neutralidad tributaria }\end{array}$ & $\begin{array}{l}\text { alto: } \\
\text { óptimo: } \\
\text { bajo: }\end{array}$ & $\begin{array}{r}0.0889 \\
0.0813 \\
-0.0548\end{array}$ & $\begin{array}{l}0.0170 \\
0.0162 \\
0.0158\end{array}$ & $\begin{array}{r}5.2280 \\
5.0182 \\
-3.4544\end{array}$ & $\begin{array}{l}0.1305 \\
0.1085 \\
0.0211\end{array}$ & $\begin{array}{l}-5.6647 \\
-5.6748 \\
-5.5462\end{array}$ & $\begin{array}{l}\text { DEBT, TOT } \\
\text { DEBT } \\
\text { FLOWS, TOT }\end{array}$ & $\begin{array}{l}57 \\
57 \\
57\end{array}$ & $\begin{array}{l}\xi^{2}{ }_{15}=15.12 \\
\xi^{2}{ }_{10}=7.95 \\
\xi^{2}{ }_{15}=17.86\end{array}$ & $\begin{array}{l}5.30 \\
5.13 \\
1.53\end{array}$ \\
\hline $\begin{array}{l}\text { TRADE: } \\
\text { Liberalización } \\
\text { comercial }\end{array}$ & $\begin{array}{l}\text { alto: } \\
\text { óptimo: } \\
\text { bajo: }\end{array}$ & $\begin{array}{r}0.0641 \\
-0.0347 \\
-0.0785\end{array}$ & $\begin{array}{l}0.0290 \\
0.0079 \\
0.0160\end{array}$ & $\begin{array}{r}2.2103 \\
-4.4177 \\
-4.9100\end{array}$ & $\begin{array}{l}0.0731 \\
0.1224 \\
0.0198\end{array}$ & $\begin{array}{l}-5.5691 \\
-5.5854 \\
-5.4748\end{array}$ & $\begin{array}{l}\text { SIGMA } \\
\text { DEBT, TOT } \\
\text { CA, FLOWS }\end{array}$ & $\begin{array}{l}52 \\
52 \\
52\end{array}$ & $\begin{array}{l}\dddot{\xi}^{2}{ }_{15}=16.60 \\
\xi^{2}{ }_{15}=18.05\end{array}$ & $\begin{array}{l}4.60 \\
2.38 \\
2.37\end{array}$ \\
\hline $\begin{array}{l}\text { SPI: } \\
\text { Promedio índices } \\
\text { sect. de políticas } \\
\text { estructurales }\end{array}$ & $\begin{array}{l}\text { alto: } \\
\text { óptimo: } \\
\text { bajo: }\end{array}$ & $\begin{array}{l}-0.1271 \\
-0.3256 \\
-0.2260\end{array}$ & $\begin{array}{l}0.0997 \\
0.0552 \\
0.1095\end{array}$ & $\begin{array}{l}-1.2748 \\
-5.9012 \\
-2.0648\end{array}$ & $\begin{array}{l}0.0487 \\
0.2162 \\
0.1357\end{array}$ & $\begin{array}{l}-5.5048 \\
-5.6984 \\
-5.6391\end{array}$ & $\begin{array}{l}\text { FLOWS, GOVSUR } \\
\text { TOT, DOCRE } \\
\text { DOCRE }\end{array}$ & $\begin{array}{l}52 \\
52 \\
52\end{array}$ & $\begin{array}{l}\xi^{2}{ }_{15}=18.08 \\
\xi^{2}=18.46 \\
\xi_{10}^{2}=16.70\end{array}$ & $\begin{array}{l}4.21 \\
3.92 \\
3.21\end{array}$ \\
\hline $\begin{array}{l}\text { SIGMA: } \\
\text { Desviación estándar } \\
\text { de los índices de } \\
\text { políticas estructurales }\end{array}$ & $\begin{array}{l}\text { alto: } \\
\text { óptimo: } \\
\text { bajo: }\end{array}$ & $\begin{array}{l}-0.2389 \\
-0.4693 \\
-0.5180\end{array}$ & $\begin{array}{l}0.1524 \\
0.0340 \\
0.0836\end{array}$ & $\begin{array}{r}-1.5673 \\
-13.8225 \\
-6.1945\end{array}$ & $\begin{array}{r}0.1137 \\
-0.0575 \\
-0.0504\end{array}$ & $\begin{array}{l}-5.6140 \\
-5.3989 \\
-5.4442\end{array}$ & $\begin{array}{l}\text { DEBT } \\
\text { CA, INF } \\
\text { FLOWS }\end{array}$ & $\begin{array}{l}52 \\
52 \\
52\end{array}$ & $\begin{array}{l}\xi_{10}^{2}=14.41 \\
\xi^{2}=18.45 \\
\xi_{10}^{2}=11.34\end{array}$ & $\begin{array}{l}2.59 \\
2.24 \\
1.40\end{array}$ \\
\hline $\begin{array}{l}\text { POLCOM: } \\
\text { SPI-SIGMA }\end{array}$ & $\begin{array}{l}\text { alto: } \\
\text { óptimo: } \\
\text { bajo: }\end{array}$ & $\begin{array}{r}0.0326 \\
-0.0723 \\
-0.0877\end{array}$ & $\begin{array}{l}0.0849 \\
0.0373 \\
0.0770\end{array}$ & $\begin{array}{r}0.3846 \\
-1.9370 \\
-1.1384\end{array}$ & $\begin{array}{l}0.0038 \\
0.1269 \\
0.0019\end{array}$ & $\begin{array}{l}-5.4971 \\
-5.5905 \\
-5.5298\end{array}$ & $\begin{array}{l}\text { GOVSUR } \\
\text { DEBT, DOCRE } \\
\text { none }\end{array}$ & $\begin{array}{l}52 \\
52 \\
52\end{array}$ & $\begin{array}{l}\xi_{10}^{2}=14.17 \\
\xi_{15}^{2}=18.65 \\
\ldots\end{array}$ & $\begin{array}{c}3.21 \\
4.89 \\
\ldots\end{array}$ \\
\hline
\end{tabular}


Bibliografía

Anderson, T.W. y C. Hsiao (1981): Estimation of dynamic models with error components, Journal of the American Statistical Association, vol.76, $\mathrm{N}^{\mathrm{o}}$ 375, Washington, D.C., Asociación Americana de Estadística, septiembre.

Arellano, M. y S. Bond (1991): Some tests of specification for panel data: Monte Carlo evidence and an application to employment equations, The Review of Economic Studies, vol. 58, № 2, Edimburgo, Society for Economic Analysis.

Aziz, J. y R. Wescott (1997): Policy Complementarities and the Washington Consensus, IMF working papers, $\mathrm{N}^{\circ} 118$, Washington, D.C., Fondo Monetario Internacional (FMI).

Baltagi, B. (1995): Econometric Analysis of Panel Data, Nueva York, John Wiley \& Sons.

Barrera, F. y E. Lora (1997): Una década de reformas estructurales en América Latina: el crecimiento, la productividad y la inversión ya no son como antes, IDB working papers, $\mathrm{N}^{\mathrm{o}} 350$, Washington, D.C., Banco Interamericano de Desarrollo (BID).

BID (Banco Interamericano de Desarrollo) (1997): Latin America after a Decade of Reforms, Washington D.C., septiembre.

Caselli, F., G. Esquivel y F. Lefort (1996): Reopening the convergence debate: A new look at cross-country growth empirics, Journal of Economic Growth, vol. 1, № 3, Dordrecht, Países Bajos.

Easterly, W., N. Loayza y P. Montiel (1997): Has Latin America's post-reform growth been disappointing?, Journal of International Economics, vol. 43, № 3/4, Amsterdam, Países Bajos, North-Holland Publishing Company.
Fernández-Arias, E. y P. Montiel (1997): Reform and Growth in Latin America: All Pain no Gain?, IDB working papers, $N^{\circ} 351$, Washington, D.C., BID.

Islam, N. (1995): Growth empirics: A panel data approach, The Quarterly Journal of Economics, vol. 110, No 4, Cambridge, Massachusetts, Harvard University.

Leamer, E. (1983): Let's take the con out of econometrics, The American Economic Review, vol. 73, № 1, Nashville, Tennessee, American Economic Association.

(1985): Sensitivity analysis would help, The American Economic Review, vol. 75, № 3, Nashville, Tennessee, American Economic Association.

Levine, R. y D. Renelt (1992): A sensitivity analysis of cross-country growth regressions, The American Economic Review, vol. 82, No 4, Nashville, Tennessee, American Economic Association.

Lora, E. (1997): Una década de reformas estructurales en América Latina: qué se ha reformado y cómo medirlo, IDB working papers, $\mathrm{N}^{\circ} 348$, Washington, D.C., BID.

Mankiw, N., D. Romer y D. Weil (1992): A contribution to the empirics of economic growth, Quarterly Journal of Economics, vol. 107, $\mathrm{N}^{\circ}$ 2, Cambridge, Massachusetts, The MIT Press.

Sala-i-Martin, X. (1997): I just ran two million regressions, The American Economic Review, Recent Empirical Growth Research, vol. 87, $\mathrm{N}^{\circ}$ 2, Nashville, Tennessee, American Economic Association.

Temple, J. (1999): The new growth evidence, Journal of Economic Literature, vol. $37, \mathrm{~N}^{\circ} 1$, Nashville, Tennessee, American Economic Association. 